Review Article

\title{
Protein Homeostasis Defects of Alanine-Glyoxylate Aminotransferase: New Therapeutic Strategies in Primary Hyperoxaluria Type I
}

\author{
Angel L. Pey, ${ }^{1}$ Armando Albert, ${ }^{2}$ and Eduardo Salido ${ }^{3}$ \\ ${ }^{1}$ Departamento de Química-Física, Facultad de Ciencias, Universidad de Granada, Avenida Fuentenueva s/n, \\ 18071 Granada, Spain \\ ${ }^{2}$ Departamento de Cristalografía y Biología Estructural, Instituto de Química Física "Rocasolano", \\ Consejo Superior de Investigaciones Cientificas, Serrano 119, 28006 Madrid, Spain \\ ${ }^{3}$ Centre for Biomedical Research on Rare Diseases (CIBERER), University Hospital of the Canary Islands, and CIBICAN, \\ University of La Laguna, 38320 Tenerife, Spain \\ Correspondence should be addressed to Eduardo Salido; esalido@ull.es
}

Received 28 April 2013; Accepted 23 May 2013

Academic Editor: Barbara Cellini

Copyright (c) 2013 Angel L. Pey et al. This is an open access article distributed under the Creative Commons Attribution License, which permits unrestricted use, distribution, and reproduction in any medium, provided the original work is properly cited.

\begin{abstract}
Alanine-glyoxylate aminotransferase catalyzes the transamination between L-alanine and glyoxylate to produce pyruvate and glycine using pyridoxal $5^{\prime}$-phosphate (PLP) as cofactor. Human alanine-glyoxylate aminotransferase is a peroxisomal enzyme expressed in the hepatocytes, the main site of glyoxylate detoxification. Its deficit causes primary hyperoxaluria type I, a rare but severe inborn error of metabolism. Single amino acid changes are the main type of mutation causing this disease, and considerable effort has been dedicated to the understanding of the molecular consequences of such missense mutations. In this review, we summarize the role of protein homeostasis in the basic mechanisms of primary hyperoxaluria. Intrinsic physicochemical properties of polypeptide chains such as thermodynamic stability, folding, unfolding, and misfolding rates as well as the interaction of different folding states with protein homeostasis networks are essential to understand this disease. The view presented has important implications for the development of new therapeutic strategies based on targeting specific elements of alanine-glyoxylate aminotransferase homeostasis.
\end{abstract}

\section{Alanine-Glyoxylate Aminotransferase and Primary Hyperoxaluria Type I}

Alanine-glyoxylate aminotransferase (AGT) is one of the aminotransferases that has raised most biomedical interest, since its deficiency causes primary hyperoxaluria type I (PH1), a rare inherited entity with unique features in terms of cellular and molecular biology of human disease. AGT, encoded by the AGXT gene, catalyzes the transamination between L-alanine and glyoxylate to produce pyruvate and glycine using pyridoxal $5^{\prime}$-phosphate (PLP) as cofactor.

As it has been the case for a number of advances in the understanding of the molecular basis of disease, the in-depth analysis of the pathogenesis of PH1 has shed light into a broader field, such as the subcellular compartmentalization of enzymes or the effect of gene modifiers on phenotype and the synergy between mutations and common genetic polymorphisms.

1.1. Role of AGT in Glyoxylate Metabolism. Glyoxylate is a two-carbon keto-acid of intermediary metabolism, with glycine, glyoxal, hydroxyproline, and glycolate as its best known sources in humans. Glyoxylate is readily converted into oxalate by various dehydrogenases and oxidases, including lactate dehydrogenase (LDH). Oxalate is an end product of metabolism in mammals that has to be eliminated with the urine; otherwise, it tends to precipitate as tissuedamaging calcium oxalate. The relevance of glyoxylate detoxification to human health is underscored by the deleterious consequences of inherited mutations in genes coding for 


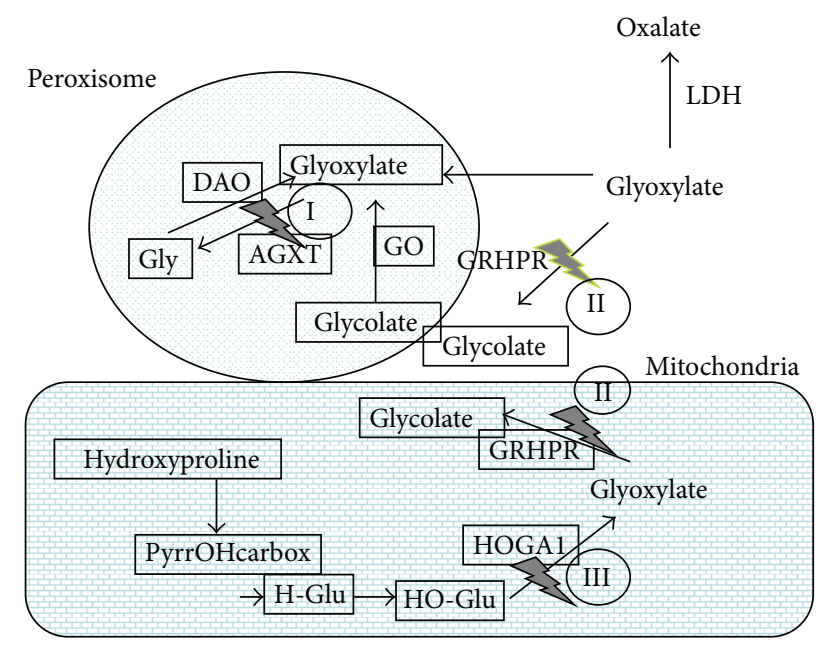

Figure 1: Summary of the glyoxylate metabolism in human hepatocytes. Simplified pathways involving glycine, glycolate, and hydroxyproline as the main sources of glyoxylate. Peroxisomal glyoxylate is detoxified by AGT, while mitochondrial and cytosolic glyoxylate is reduced to glycolate by GRHPR, preventing excessive oxidation to oxalate by LDH. Hydroxyproline metabolism results in the production of 4-hydroxy-2oxoglutarate that is normally split into glyoxylate and pyruvate by HOGA1. PyrrOHcarbox=pyrroline-5-carboxylate; HGlu $=4$ hydroxy-glutamate; $\mathrm{HO}-\mathrm{Glu}=4$-hydroxy-2-oxoglutarate. The three genetic defects currently known to cause $\mathrm{PH}$ are inherited with autosomal recessive pattern. The genes involved are alanine-glyoxylate aminotransferase (AGXT, at $2 \mathrm{q} 37.3, \mathrm{MIM} * 604285)$, for PH type I (PH1, MIM\#259900), glyoxylate reductase/hydroxypyruvate reductase (GRHPR, at 9q12, MIM*604296), for $\mathrm{PH}$ type II (PH2, MIM\#260000), and 4-hydroxy-2-oxoglutarate aldolase (HOGA1 at $10 \mathrm{q} 24.2, \mathrm{MIM} * 613597)$, for PH type III (PH3, MIM\#613616).

key enzymes in this pathway, AGXT being one of them (Figure 1). Human conditions characterized by high oxalate levels in urine are known as hyperoxalurias, and their genetic forms, termed primary hyperoxalurias $(\mathrm{PH})$, are due to high oxalate production by hepatocytes deficient in one of these enzymes [1-3]. PH patients have urinary excretion levels $>0.5 \mathrm{mmoL} / 1.73 \mathrm{~m}^{2}$ per day (typically $>1 \mathrm{mmoL} / 1.73 \mathrm{~m}^{2}$ ), while normal oxalate excretion is below $0.45 \mathrm{mmoL} / 1.73 \mathrm{~m}^{2}$.

Since LDH is abundant in the hepatocyte cytosol and vertebrates do not have a functional glyoxylate shunt capable of using glyoxylate as a substrate for the tricarboxylic acid cycle, most of the glyoxylate generated must be metabolized within organelles such as the peroxisome and mitochondria in order to limit oxalate production. To further control the levels of oxalate produced, cytosolic glyoxylate reductase (GRHPR) competes with LDH for glyoxylate, reducing it to glycolate, a highly soluble two-carbon molecule.

Glyoxylate detoxification reflects the evolutionary origins of metabolic partitioning into the various subcellular organelles [5]. Thus, the subcellular distribution of the key enzymes of the glyoxylate detoxification pathway has been under evolutionary pressure and diet must have been an important component of such pressure, since glycolate is abundant in vegetables while hydroxyproline is abundant in meat.

Human AGT is a hepatocyte-specific enzyme that is normally located in the peroxisomes only [6], making this organelle an efficient site for detoxification of glyoxylate either imported from the cytosol or mitochondria or produced in situ by either D-amino acid oxidase (DAO) or hydroxyacid oxidase (HAOl) (glycolate oxidase), using glycine or glycolate as substrate, respectively. The peroxisome membrane is permeable to glycolate, glyoxylate, and other small hydrophilic solutes, largely through the PXMP2 channel [7]. Since AGT can tolerate high glyoxylate concentrations [8], the peroxisome, rich in AGT, plays a crucial role as glyoxylate detoxifying compartment that shields the surrounding cytoplasm from glyoxylate accumulation and secondary oxalate production.

Mitochondria also play an important role in glyoxylate metabolism $[9,10]$. In humans, this role is based on their capacity to metabolize hydroxyproline [11], but in mammals with mitochondrial AGT this enzyme is also central to glyoxylate detoxification in this organelle. Collagen, containing $\sim 15 \%$ hydroxyproline, is a major constituent of extracellular matrix and daily collagen turnover yields $300-$ $450 \mathrm{mg}$ hydroxyproline, accounting for the production of $180-240 \mathrm{mg}$ glyoxylate $[12,13]$. The last step of this pathway involves the cleavage of 4-hydroxy-2-oxoglutarate (2keto-4-hydroxyglutarate) into glyoxylate and pyruvate by $4-$ hydroxy-2-oxoglutarate aldolase (HOGAl). The glyoxylate can then be converted to glycolate by GRHPR.

1.2. Primary Hyperoxaluria Type I. $\mathrm{PH}$ has an estimated prevalence of 1-3 per million population and an estimated incidence rate of $\sim 1: 100,000$ live births per year in Europe [14-16], although the exact prevalence is unknown due to underdiagnosis. The most comprehensive attempts to estimate the true incidence of the disease [16] have resulted in higher incidence rates than previously reported. Higher rates have also been found in historically isolated populations, like the Canary Islands, due to founder effect [17]. Although $\mathrm{PH}$ accounts for less than $1 \%$ of children in end-stage renal disease (ESRD) registries of developed countries [18], almost $10 \%$ of Kuwaiti children and $13 \%$ of Tunisian children with ESRD have been reported to suffer PH $[19,20]$.

$\mathrm{PH} 1$, caused by deficient or mistargeted AGT [21], is the most common (around 80\%) and the most severe $\mathrm{PH}$ type, usually resulting in ESRD at some point, although with a wide range of severity. At ESRD, the buildup of oxalate in the body (known as oxalosis) quickly results in bone, heart, skin, and retinal complications. Oxalosis is a life-threatening condition, unless liver-kidney transplantation is performed $[14,17,22]$.

The interest in $\mathrm{PH} 1$ prompted the cloning of AGXT cDNA $[23,24]$, using probes from the orthologous rat gene [25]. The gene has 11 exons and spans $\sim 10 \mathrm{~kb}$ [26], resulting in a $1.7 \mathrm{~kb}$ mRNA with a coding sequence of $1,176 \mathrm{bp}$. The gene product AGT is a homodimeric protein, each $43 \mathrm{kDa}$ subunit containing 392 amino acids and holding one molecule of PLP as cofactor [27]. The main N-terminal domain contains 
most of the catalytic active site, the cofactor-binding site, and the dimerization interface. The smaller C-terminal domain is known to interact with the peroxisomal receptor PEX5, targeting the dimer to the peroxisome. AGT, carrying a noncanonical peroxisomal targeting sequence (PTS1), is among the peroxisomal proteins with the weakest affinities for PEX5 [28]. An ancillary sequence surrounding amino acids 324345 has been proposed to help the peroxisomal targeting of AGT [29]. A recently released crystal structure of the AGT in complex with the PTS1-binding domain of PEX5 (PDB: 3IMZ) also confirmed that residues 303-306 and 327-330 are largely buried upon binding. In fact, AGT binds to PEX5 with $\sim 10$-fold higher affinity than its PTS1 octapeptide, showing the functional role of this ancillary sequence in PEX5 binding [30].

The 3D structure of AGT (PDB: 1H0C) [27] has provided important information to better understand the function of the protein and the effect of changes in amino acid that account for a majority of the PH1 mutations (see Section 3 below).

More than 150 mutations have been described for AGXT, and they have been summarized recently [31]. Missense mutations are common, followed by small insertion/deletions (indels). Wild type AGXT comes in two polymorphic variants, the most frequent major haplotype (refseq NM_000030) and the less frequent minor haplotype, carrying two single amino acid substitutions (p.P11L and p.I340M) among other genomic changes in strong linkage disequilibrium. Since these two polymorphisms are quite old, most of the individual PH1 mutations described are typically found in either the major or minor haplotype, but rarely in both, which is useful when searching for mutations in new PH1 cases [32]. The minor haplotype (simply defined by the refSNP rs34116584, p.ProllLeu), with an allelic frequency of 0.1-0.2 in western countries and average heterozygosity around 0.2 , does not cause $\mathrm{PH} 1$ by itself, but it is known to act synergistically with the deleterious effects of several common mutations $[33,34]$.

1.3. Molecular Mechanisms of Disease. AGXT mutations result in severe reductions of AGT enzymatic activity in the peroxisome, with a relatively wide range of residual activity, depending on the mutations present in both alleles [35]. Although AGT functions as a dimer, all the mutations described so far are related to loss of function, with recessive pattern of inheritance, without evidence of potential dominant negative effect.

Small indels are responsible for some PH1 cases due to AGT synthesis defects, most notably c.33dupC, the main mutation of the major haplotype, with the predicted consequence of early stop codon and nonsense mediated mRNA decay. We could also include in this category of synthesis defects most splicing mutations and occasional missense mutations leading to highly unstable protein that is degraded rapidly, such as p.S205P [36]. But the majority of $\mathrm{PH} 1$ alleles are missense mutations, with four potential molecular mechanisms involved: mitochondrial mistargeting, protein aggregation, catalytic defects, and enhanced turnover.
About a third of PH1 alleles involve the p.G170R substitution, in the minor haplotype, which is responsible for mitochondrial mistargeting of the gene product, becoming one of the best known examples of human mutations resulting in mistargeting as the main mechanism of disease [37-39]. In addition to p.G170R, p.F152I, also in the minor haplotype, was found to cause AGT mistargeting to the mitochondria instead of the peroxisome, depleting the latter organelle of its glyoxylate detoxifying capability.

The polymorphism p.P11L of the minor haplotype plays a crucial role for p.G170R to result in mitochondrial mistargeting [40], which has been associated to impaired folding efficiency to form functional dimers, therefore allowing mitochondrial import of mutant AGT [41].

Protein aggregation is a relatively frequent outcome of missense mutations in conformational diseases [42]. Several of the most frequent PH1 mutations of the minor haplotype, such as p.G41R [37] and p.I244T [34], are known to display protein aggregation. The p.P11L polymorphism was also found to play a crucial role for the I244T mutation to result in a conformationally unstable protein, prone to aggregation [34], while the p.G41R mutation disturbs the local interactions of the $\mathrm{N}$-terminus, causing conformational instability as well [43].

Catalytic defects are a common mechanism of disease in inborn errors of metabolism involving enzyme-coding genes. Enzymatic characterization of several PH1 missense mutations (p.G82E, p.G41R, and p.F152I) have demonstrated significant alterations in their performance, including strong decreases in catalytic efficiency and reduced binding affinities for PLP and PMP cofactors [44].

Genotype-phenotype correlations have been described for some mutations of the AGXT gene [33, 45, 46], but the wide allelic heterogeneity limits this type of analysis to the most common mutations, unless large international registries are used. Significant environmental influences and the potential effect of genetic modifiers also play an important role to the point that siblings who share the same genotype could have very different clinical phenotypes [47].

\section{Structure-Function Relationships of AGT}

The crystal structure of AGT was solved at atomic resolution [27] to gain insights on the molecular and structural basis of the disease. AGT is a homodimer with each protomer folded into a large $\mathrm{N}$-terminal domain (residues 22-282) and a smaller C-terminal domain (283-392). Most of the contacts within the dimer involve the large $\mathrm{N}$-terminal domain. Besides, a long unstructured N-terminal tail (residues 1-21) grabs the subunits within the dimer (Figure 2(a)). This structure provided valuable information about the enzyme functioning and a framework to map the mutations of the AGT gene. This analysis showed that they are almost randomly scattered over the entire three-dimensional structure of the enzyme [30], making it difficult to establish general rules on the molecular consequences of these variants. However, the 


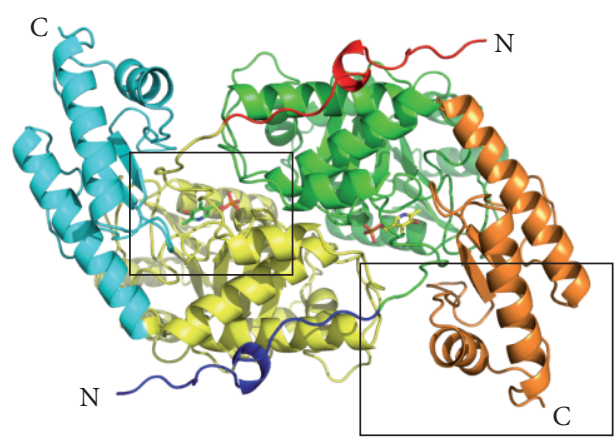

(a)

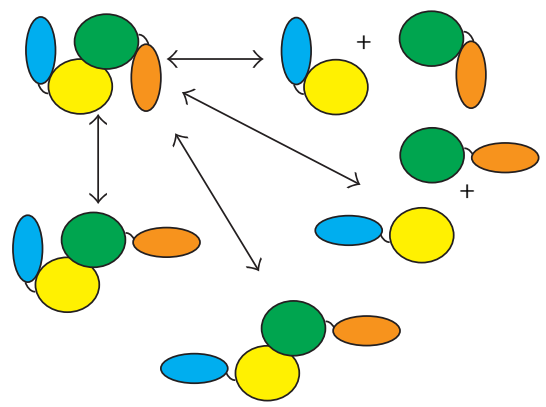

(c)

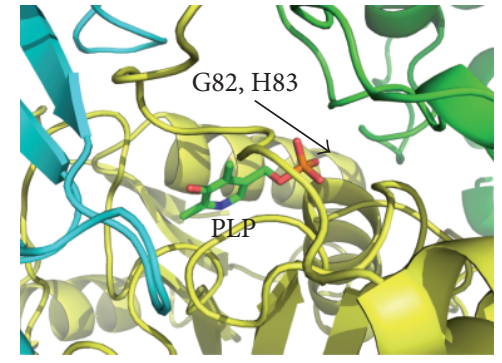

(b)

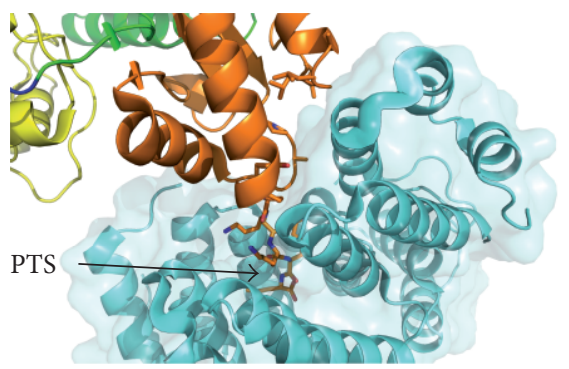

(d)

FIGURE 2: The structure of AGT. (a) A ribbon representation of the dimeric AGT structure (PDB code 1H0C) colored to highlight the domain organization. The black squares represent the zoomed sections shown in panels $1 \mathrm{~b}$ and $1 \mathrm{~d}$. (b) A representation of the PLP binding site; G82 and H83 are in the vicinity of the cofactor. (c) A schematic representation of the possible "almost folded" AGT intermediates. (d) A representation of the AGT Pep5x intermolecular interface. The molecular surface representation of Pex5p is also displayed and colored in cyan. Those AGT residues interacting with Pep5x are displayed in a stick representation.

available structural information resolves central questions on the knowledge about the basis of the disease.

The effect of mutations affecting the enzymatic properties of AGT can be rationalized in structural terms. p.G82E and p.H83R are well characterized to produce catalytic defects $[8,48]$. The analysis of the structure reveals that both of them cluster in the vicinity of the active site. These changes involve bulkier side chains that hinder either cofactor or substrate binding (Figure 2(b)). By contrast, the attempts to rationalize the effect of variants leading to protein aggregation in terms of the structure have been unsuccessful since it is likely that either kinetic and/or thermodynamic changes are the main mechanism involved [48]. The modeling of these mutations on the structure reveals no significant changes since they often involve either conservative changes or solvent accessible residues. Consequently, it is not expected to induce large predictable conformational changes in AGT structure. This is well illustrated by the structural analysis of the G170R variant, which resulted in a nearly identical structure to the wild type [49]. In addition, the expression of many of these mutations yields unstable aggregated and/or partially unfolded products that cannot be crystallized.

The three-dimensional structure of the complex between the ring chaperonin GroEL and AGT-LTM variant provided evidence that the mutated enzyme is able to form nonnative folding intermediates that interact with the chaperone [50].
These intermediates consist of an "open" version of AGT protomer in which the small and the large domains are correctly folded (Figure 2(c)). Such intermediates may be prone to protein aggregation but they constitute a promising target for pharmacological chaperones.

The crystal structure of the AGT in complex with the PTS1-binding domain of Pex5p was determined to shed light into the mechanism of AGT import into peroxisomes [30]. The complex displays Pex5p-AGT-AGT-Pex5p stoichiometry and confirms that dimeric and perfectly folded AGT interacts with the receptor. On the AGT side, the complex buries completely those residues forming the PTS1 and some constituting the smaller C-terminal domain (Figure 2(d)). This suggests that those mutations affecting the surface properties of the interaction area will affect the import to peroxisomes.

\section{Biophysical, Biochemical, and Cell Biology Approaches to Study AGT Deficiency and Protein Homeostasis Defects}

Protein homeostasis controls the functional properties of proteins by minimizing the presence of misfolded protein states that may be damaging to cellular function [51,52]. A complex and highly interacting and regulated network of pathways, involving over 800 different proteins, is in charge of protein homeostasis [53], regulating protein synthesis, 


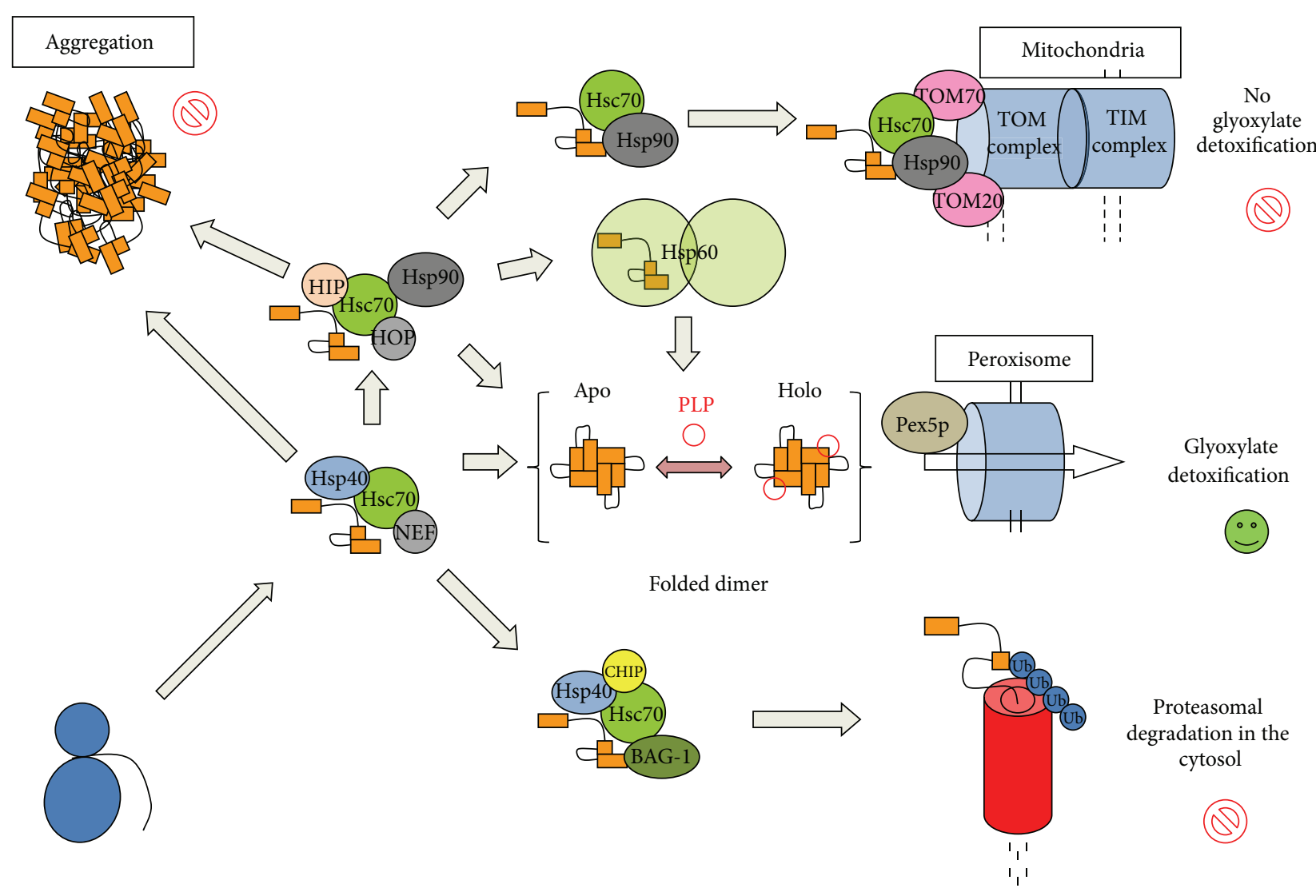

FIGURE 3: A simple scheme representing potentially important checkpoints in the folding and misfolding of human AGT. After ribosomal synthesis, the AGT monomer is maintained in a partially folded and soluble state upon interaction with Hsp40 chaperones allowing its engagement to the Hsp70 machinery. Correct folding may proceed through the transfer of the partially folded polypeptide to the Hsp90 and Hsp60 machineries leading to the folded holo-AGT dimer and peroxisomal import through the Pex5p import machinery. However, PH1 causing mutants to show enhanced interactions with Hsp70, Hsp60, and Hsp90 chaperone systems which may (i) delay correct folding eventually causing AGT aggregation; (ii) allow engaging the proteasomal degradation machinery mediated by CHIP and BAG-1 proteins; (iii) allow Hsp70/Hsp90 mediated presentation to the mitochondrial import machinery via TOM20 or TOM70 receptors. For further details and references, see the main text.

folding, trafficking, and degradation $[51,52]$. Protein homeostasis defects are associated with aging and disorders of protein folding, including metabolic diseases, cancer, and neurodegenerative diseases $[51,52,54]$. In the context of protein homeostasis, intrinsic physicochemical properties of polypeptide chains such as thermodynamic stability, folding, unfolding, and misfolding rates as well as the interaction of different folding states with protein homeostasis networks are essential to understand protein folding and misfolding under physiological and pathological conditions [54]. In this section, we summarize the knowledge on protein homeostasis defects in $\mathrm{PH} 1$ to provide a comprehensive and integrated perspective of $\mathrm{PH} 1$ as a folding disease. The view presented has important implications for the development of new therapeutic strategies for $\mathrm{PH} 1$ based on targeting specific elements of AGT protein homeostasis (summarized in Figure 3).
3.1. Stability of AGT Variants toward Chemical Denaturants, Temperature, and Proteases: Mechanistic Implications. Several studies have addressed the unfolding of AGT, both wild type (WT) and PH1 mutants, by either chemical denaturants (guanidine, urea, and $\mathrm{pH}$ ) [55-57] or temperature [44, 48, 56, 57]. The mechanistic studies derived from these studies are discussed in this section in some detail.

\subsubsection{Chemical Denaturation of AGT WT and Disease Caus-} ing Variants: Presence of Unfolding Intermediates. Chemical unfolding by guanidium, urea, and mild acidic $\mathrm{pH}$ has been shown to irreversibly denature AGT at the experimental conditions used by different research groups [55-57]. We must note that the term irreversible is used here to indicate that removal of the denaturant does not provide the refolding yields required for applying equilibrium thermodynamic 
analyses. However, we must also note that very mild refolding conditions have proved to enhance remarkably AGT refolding yields [58]. Therefore, we will not attempt to extract thermodynamic information from the biophysical studies discussed here, even though they may provide insight into partially folded states that could be relevant to understand the (un)folding pathways of AGT variants in vitro (and possibly intracellularly), the effect of PH1 mutants on folding of AGT and the role of molecular chaperones in AGT folding and misfolding.

Unfolding of holo-and-apo AGT WT, minor AGT (LM, p.P11L-I340M) and LM-G170R (p.G170R in minor AGT LM-, LRM for short) variants by guanidium hydrochloride and mild acidic $\mathrm{pH}$ have revealed the presence of a moltenglobule-like unfolding intermediate (MG). This MG intermediate does not refold to the native state spontaneously and is able to interact with molecular chaperones in cellfree systems, suggesting that last folding steps of AGT may require help from molecular chaperones in vitro and intracellularly [57]. Interestingly, Hopper et al. [56] have shown that native state ligands such as PLP and AOA stabilize AGT $\mathrm{WT}$ and LM towards guanidium denaturation, even though the thermodynamic or kinetic basis of such stabilization and their relation with the partially unfolded states are unclear. Aggregation-prone unfolding intermediates are also observed in the urea induced denaturation of AGT, especially in the apo-forms and/or when the P11L polymorphism is present [55]. Indeed, the partially unfolded states found in the unfolding pathways of LRM variant might be related to its tendency for mitochondrial import [55], also explaining the strong interaction of this variant along its folding process with Hsp70 and Hsp90 chaperones in cell-free systems [57]. Additionally, aggregation of apo-AGT WT in refolding trials is prevented by the presence of the bacterial Hsp40 DnaJ in a concentration dependent manner (Figure 4), suggesting that binding to this chaperone is the first step to engage the Hsp70 machinery (Figure 3).

3.1.2. Thermal Denaturation of AGT Variants: The Role of AGT Kinetic Stability in PH1. Thermal denaturation of AGT variants has been monitored by multiple techniques, including activity, circular dichroism, differential scanning fluorimetry (DSF), and differential scanning calorimetry (DSC) $[43,44,48,55-57,59,60]$. All these studies have shown that withdrawal of PLP has a dramatic effect in terms of stability, reducing $20-25^{\circ} \mathrm{C}$ AGT thermal stability. The denaturation of holoproteins is consistent with a single denaturation transition using multiple probes, while denaturation of apoproteins is sometimes described by a one or two thermal transitions, depending on the AGT variant and the technique monitoring denaturation $[48,55-57,59,60]$. The presence of two denaturation events has been discussed as the uncoupled denaturation of the large and small domains [59]. However, we must note that enzyme inactivation coincides with the low $T_{m}$ transition found by Far-UV CD and DSF $[48,56,59,60]$ and the single transition found by DSC $[48,57]$. Moreover, kinetic analyses of DSC transitions based on a two-state denaturation model provide denaturation rates at $37^{\circ} \mathrm{C}$ for apoproteins in excellent agreement with those obtained from inactivation kinetics, indicating that DSC based kinetic analyses monitor irreversible denaturation and inactivation of apo-proteins [48]. Almost all studies coincide in the destabilizing effect of the minor allele, while additional mutations further reduce AGT thermal stability $[44,48,56$, $57,59,60]$.

We have recently performed a thorough characterization of thermal denaturation of holo- and apoproteins by differential scanning calorimetry in AGT WT and eleven mutants and polymorphic variants found in PH1 patients $[48,57]$. Denaturation of all AGT variants as apo- and holoproteins is described by a phenomenological two-state irreversible denaturation model. In this scenario, the stability of the native state is determined by the rate of irreversible denaturation $k$, that relates to the half-life for denaturation by $t_{1 / 2}=\ln 2 / k$ $[2,48,57]$. The value of $k$ is determined by the height of the activation free energy that the native state must cross to reach the denaturation (rate-limiting) transition state (TS) $[48,57,61]$. Thermal denaturation of AGT mostly involves a dimeric TS, indicating that the kinetic stability of AGT enzymes is mainly dictated by the impact of mutations on the free energy of the dimeric native and TS states. Thus, dimer dissociation and monomer unfolding do not contribute to the AGT kinetic stability, because these steps must occur after the denaturation rate-limiting step [48]. The large kinetic stabilization exerted by PLP results from its preferential binding to the native state, increasing the denaturation free energy barrier by $\sim 7 \mathrm{kcal} \cdot \mathrm{moL}^{-1}[48,57]$. The temperature dependence of calorimetric enthalpies agrees well with the theoretical value for a dimer of this size, suggesting that holo- and apo-proteins unfold extensively and to a similar extent upon thermal denaturation, and possibly involving denaturation of both domains in AGT $[48,57]$.

Kinetic analyses of DSC traces provide a unique opportunity to compare denaturation rates among AGT variants spanning a wide range of stabilities (half-lives from minutes to years at physiological temperature). For instance, removal of PLP decreases (in WT and most of PH1 variants) AGT kinetic stability by $4-5$ orders of magnitude $[48,57]$. The presence of P11L and LM polymorphism reduces AGT kinetic stability by $\sim 150$ and $\sim 20$-fold, while the presence of I340 M kinetically stabilizes AGT compared to AGT WT (Fabelo-Rosa, submitted). In general, PH1 causing mutants show similar stability to AGT LM as holo-proteins, with a remarkably high kinetic stability (half-lives in the range of years at $37^{\circ} \mathrm{C}$ ), while they often reduce apo-AGT kinetic stability (and denature in a time scale of minutes-hours; $[48,57,62])$. Thus, PLP binding overstabilizes the AGT native state in some mutants [2] which might explain the PLP responsiveness found for some of them in $\mathrm{PH} 1$ patients [63, 64]. Therefore, targeting the cellular systems responsible for PLP bioavailability (enzymes involved in PLP recycling and delivery; [65]) may represent a pharmacological approach to overcome mutation induced protein destabilization in PH1.

3.1.3. Resistance to Proteolysis: Effects of PH1 Mutations on Protein Flexibility and High-Energy States. Proteolysis is an 


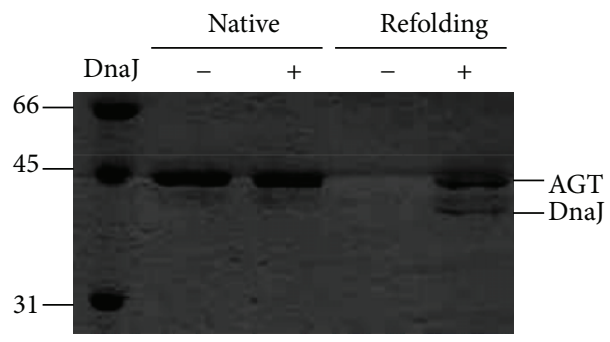

(a)

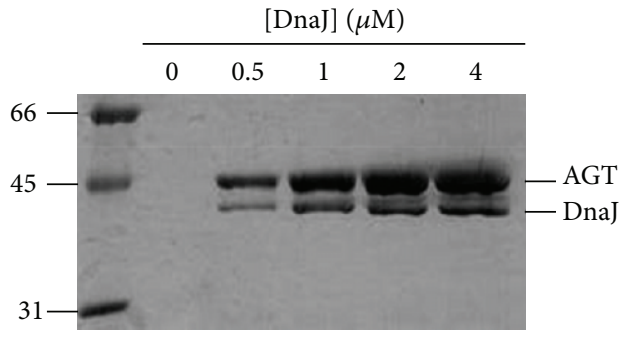

(b)

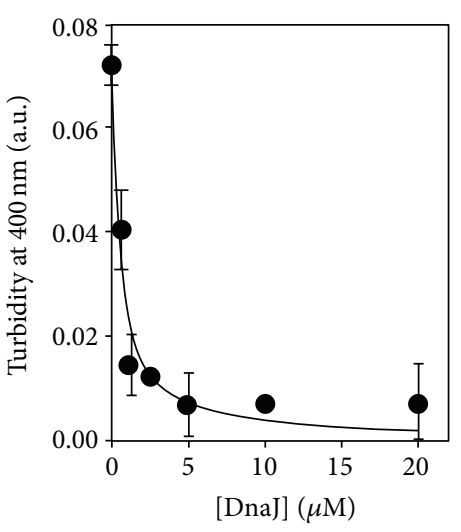

(c)

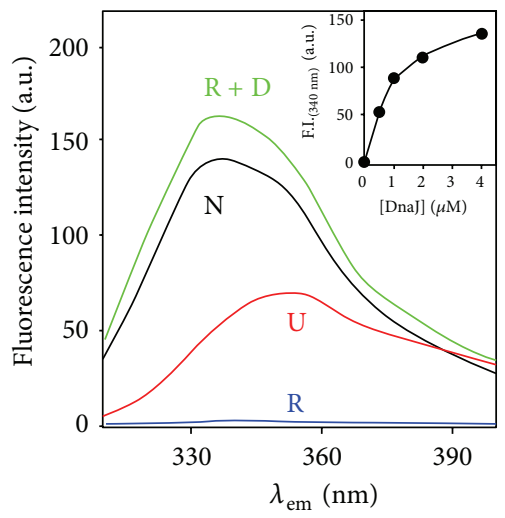

(d)

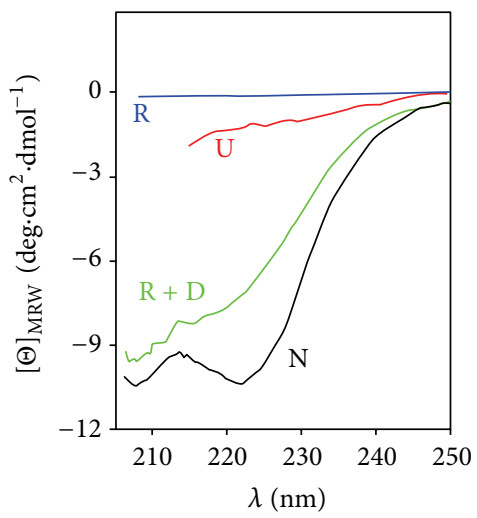

(e)

FIGURE 4: DnaJ prevents aggregation of a partially folded state of AGT. (a) IMAC-based copurification assay of his-tagged apo-AGTwt with DnaJ $(1 \mu \mathrm{M}$ each), under native (no urea) and refolding (20-fold dilution from a $16 \mathrm{~h}$ incubated sample with $8 \mathrm{M}$ urea) conditions; (b) DnaJ protein concentration dependence of its interaction with apo-AGTwt under refolding conditions (the same conditions as in (a)). (c) DnaJ protein concentration dependence of the maximal turbidity at $400 \mathrm{~nm}$ in the apo-AGTwt refolding (data are from three independent experiments); (d) and (e) solubility and conformational assays of Apo-AGTwt based on its intrinsic Trp-emission fluorescence ((d); exc.295 nm) or Far-UV CD (e) under different conditions: native (0 M urea; N), unfolded (8 M urea; U), and apo-AGTwt refolded in the absence $(\mathrm{R})$ or presence of $4 \mu \mathrm{M}$ DnaJ $(\mathrm{R}+\mathrm{D})$. After urea-dilution, samples were incubated at $25^{\circ} \mathrm{C}$ for $30 \mathrm{~min}$, centrifuged at $15000 \mathrm{rpm}$ for $30 \mathrm{~min}$, and the spectroscopic analyses were performed in the supernatants. The contribution from DnaJ to fluorescence is negligible due to the absence of Trp residues, while its contribution to Far-UV-CD spectra was subtracted from R + D. Inset: DnaJ protein concentration dependence of the Trp-fluorescence on the soluble fraction. A fitting to a hyperbolic function is shown, providing half-maximal fluorescence recovery at $1.0 \pm 0.1 \mu \mathrm{M}$ DnaJ. All the experiments were performed at $25^{\circ} \mathrm{C}$ in Na-Hepes $20 \mathrm{mM} \mathrm{NaCl} 200 \mathrm{mM} \mathrm{pH} 7.42 \mathrm{mM}$ DTT using $1 \mu \mathrm{M}$ AGT (in protein subunit). DnaJ was purified according to [4].

excellent method to study protein flexibility and transient population of protein high energy states, as long as flexible or partially unfolded regions in the protein are accessible for binding and proteolytic cleavage [66]. Indeed, kinetics of proteolysis has provided information on protein high energy states present in native state ensembles which are not accessible for most of ensemble averaged-based biophysical methods [66]. Several studies have addressed the stability of AGT enzymes towards proteolysis using different proteases $([34,43,57,67,68]$ and Figure 5). Trypsin and proteinase $\mathrm{K}$ degradation have been applied to several $\mathrm{PH} 1$ mutants [34, 43, 68], showing that P11L polymorphism enhances protease sensitivity that is further exacerbated by additional mutations. In the case of LM-G41R, detailed characterization of proteolysis products in combination with molecular dynamic simulations supports that G41R mutation enhances conformational fluctuations in the N-terminal region of AGT, thus accelerating cleavage by proteases $[43,68]$. Interestingly, native state ligands and naturally occurring osmolytes are able to increase protease resistance in several PH1 mutants [68], suggesting that reshaping of the native state ensemble energetics by ligands may modulate the conformational fluctuations in some PH1 mutants. From a physiopathological viewpoint, studies in cell free systems on the proteasomal susceptibility also reveal that disease causing mutations enhance degradation [67].

AGT WT undergoes rapid cleavage by thermolysin leading to an active $\sim 75 \mathrm{kDa}$ dimer (referred here to as $\mathrm{D}^{*}$ state) [57]. The stability of this $\mathrm{D}^{*}$ state has been further characterized by a combination of thermolysin digestion and thermal scans for different holo-AGT enzymes (Figure 4). The apparent rate constants for proteolysis are extracted from 


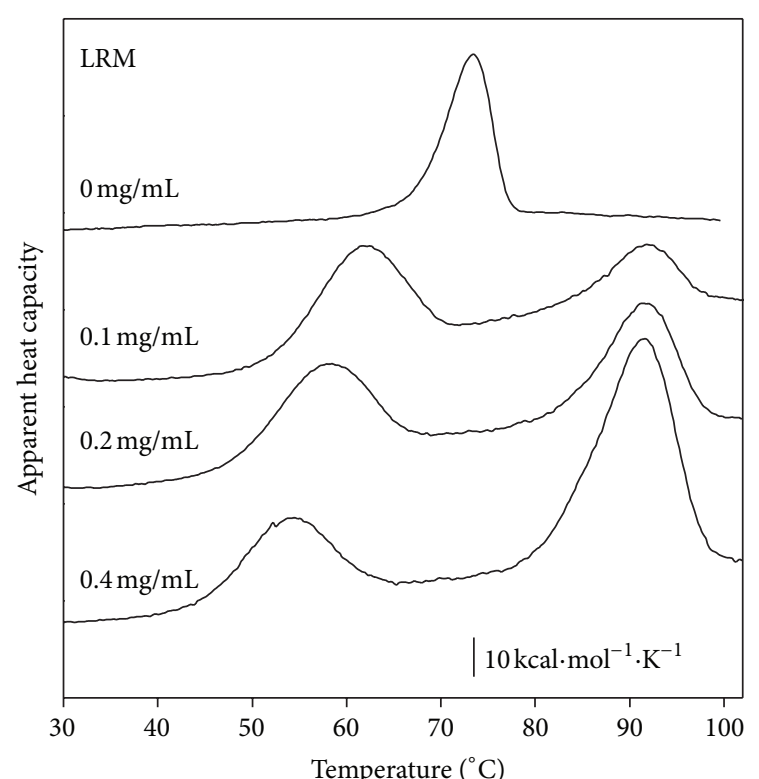

(a)

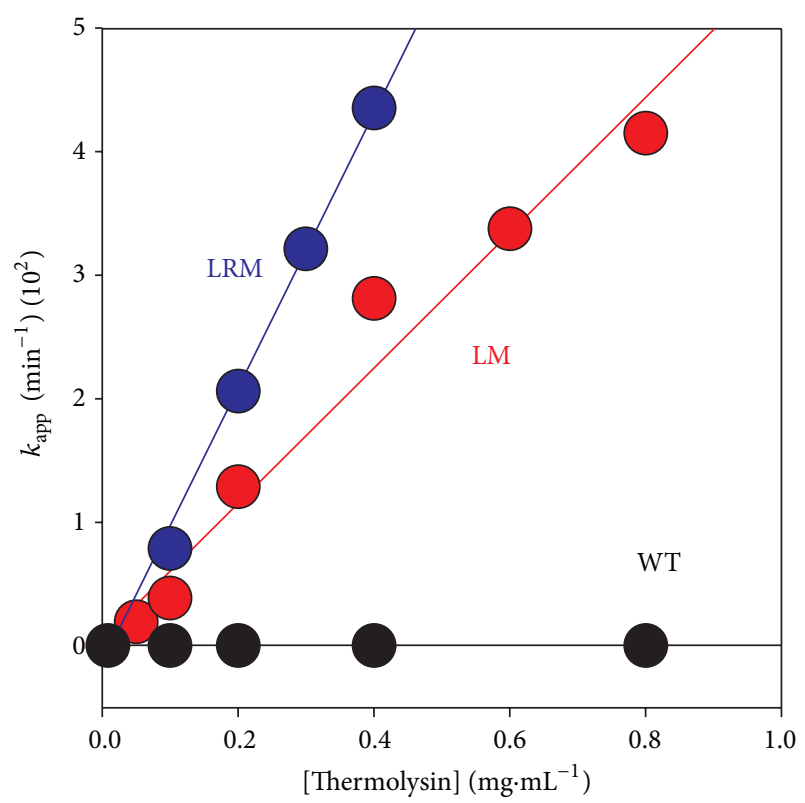

(c)

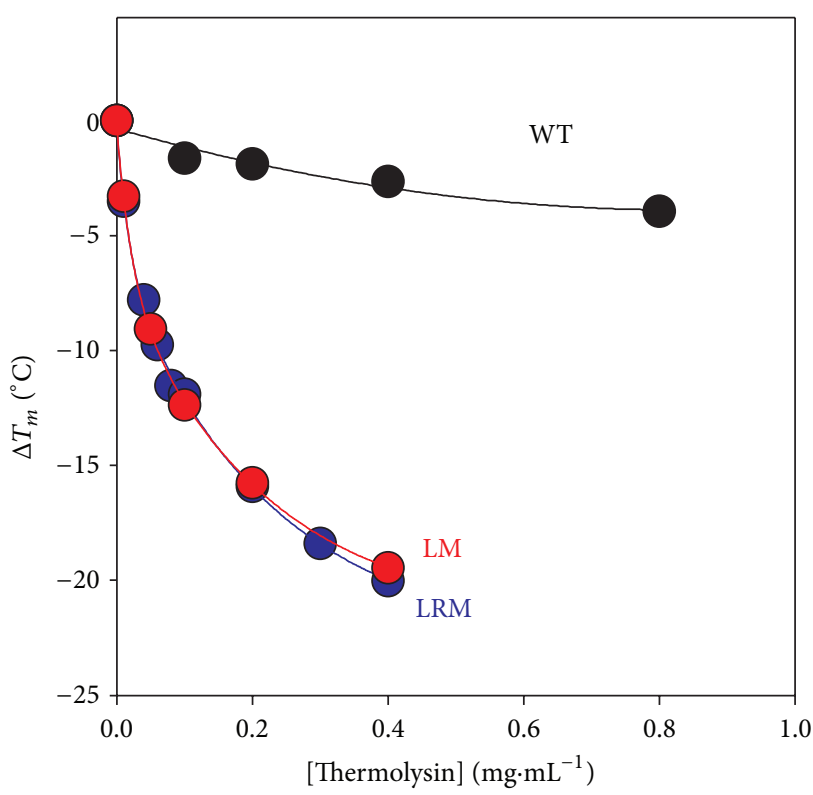

(b)

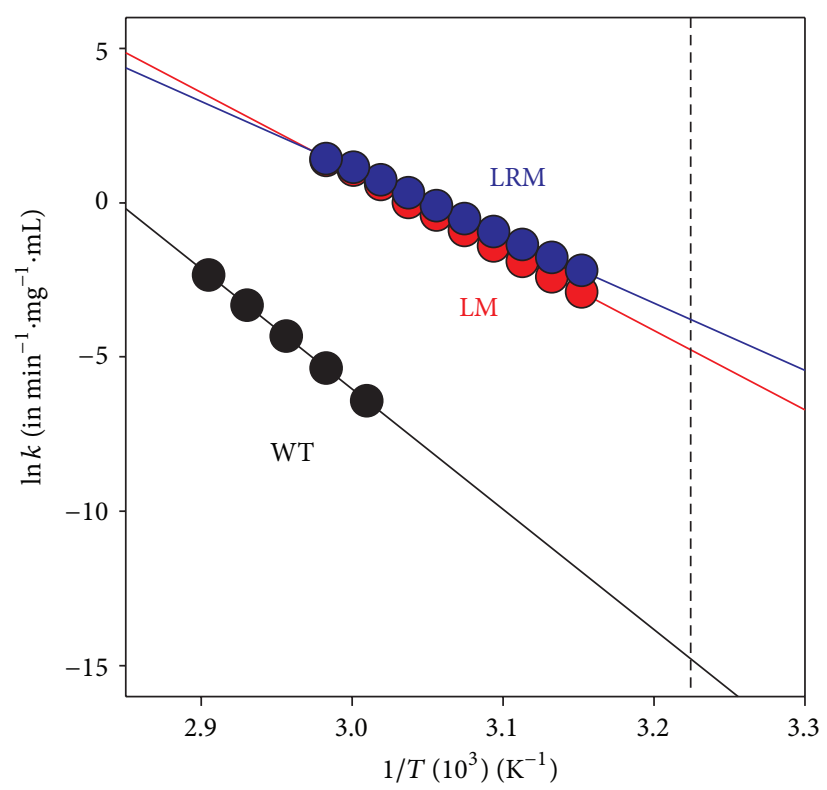

(d)

FIGURE 5: Temperature dependent proteolysis of AGT variants by thermolysin. (a) thermal scans of AGT-LRM (10 $\mu$ M in protein subunit) in the presence of different thermolysin concentrations $(0-0.4 \mathrm{mg} / \mathrm{mL})$. The high temperature transition $\left(T_{m} \sim 90^{\circ} \mathrm{C}\right)$ corresponds to thermolysin denaturation, which is thermostable. (b) Dependence of $T_{m}$ downshift of thermal transitions of AGT variants by thermolysin. (c) Dependence of the apparent proteolysis rate constants on thermolysin concentration at $44^{\circ} \mathrm{C}$. The slope of these plots provides the secondorder rate constants (plotted in (d)); (d) Arrhenius plots for the second-order rate constants for proteolysis. The vertical dashed line indicates $37^{\circ} \mathrm{C}$. Experiments were performed in Hepes $20 \mathrm{mM} \mathrm{NaCl} 200 \mathrm{mM} \mathrm{CaCl} 210 \mathrm{mM}$.

thermal scans at a given protease concentration in the temperature range of the transition [69]. The $T_{m}$ value of LM and LM-G170R is shown to be more sensitive to the concentration of protease than that of WT AGT (Figure 4(b)).The apparent first-order rate constants for proteolysis (Figure 4(c)) indicate that proteolysis is $\sim 6$ orders of magnitude slower for WT AGT than for the LM and LM-G170R, while LM-G170R is degraded about twice faster than AGT LM. At $37^{\circ} \mathrm{C}$ (Figure $4(\mathrm{~d})$ ), the half-lives against proteolysis (at $0.1 \mathrm{mg} / \mathrm{mL}$ protease) are 60-(WT), 3.9.10 ${ }^{4}$-(LM), and 6.2 $10^{4}$-fold (LMG170R) lower than those determined in the absence of protease, suggesting that holo-AGT enzymes might be very sensitive to proteolysis despite their robustness towards thermal induced aggregation. 
3.2. Protein Homeostasis of AGT: Implications to Develop New Therapeutic Strategies. Peroxisomal import of human AGT occurs upon direct interaction of the fully folded dimer with Pex5p in the cytosol. Multiple lines of evidence support that protein folding defects are implicated in AGT loss of function in $\mathrm{PH} 130,48]$. However, dissecting the protein homeostasis defects in PH1 represents a remarkable challenge, since the biomolecular interactions of partially folded states with the protein homeostasis network may involve over 200 different proteins in cytosolic folding ([53]; see Figure 3 for a very simplistic view in $\mathrm{PH} 1)$. In this section, we do our best to gather the most relevant available information on the physiological folding and peroxisomal targeting, as well as those protein homeostasis defects alterations that may lead to AGT loss of function due to different mechanisms, including mitochondrial mistargeting, enhanced protein aggregation, and degradation.

3.2.1. Peroxisomal and Mitochondrial Targeting of Human AGT. The PTS1 of human AGT is suboptimal (-KKL versus the consensus-SKL; [41]), and its molecular recognition by Pex 5 requires additional structural elements at the Cterminal domain $[29,30]$. In fact, the interaction of AGT WT and Pex5p PTS1 binding domain (Pex5p-pbd) is of moderate affinity $\left(K_{d} \sim 1.5-3.5 \mu \mathrm{M} ;[30,48]\right)$ and decreases 10 -fold for the isolated C-terminal PTS1 octapeptide [2, 28]. The low affinity of human AGT for its peroxisomal receptor may have several important implications to understand the evolutionary changes in AGT subcellular location and function $[70,71]$, the correct peroxisomal biogenesis [28], and the role of peroxisomal import in $\mathrm{PH} 1$ pathogenesis $[30,48]$.

AGT subcellular location seems to vary among species depending on a delicate balance between mitochondrial and peroxisomal import signaling pathways and the capacity of cytosolic protein homeostasis networks to fold AGT proteins $[70,71]$. The presence of mitochondrial AGT in carnivores, peroxisomal AGT in herbivores, and both locations in omnivores suggest that subcellular location of AGT may have resulted from dietary selection pressure [72]. Additionally, the presence of a strong N-terminal mitochondrial targeting sequence (MTS) in AGT from X. laevis drives the mitochondrial localization of this enzyme, while the corresponding N-terminal MTS seems to be weak in human AGT [71]. The weak MTS in hAGT seems to be strenghtened by additional natural variations associated with mitochondrial mistargeting, such as P11L polymorphism and the G170R, F152I and G41R mutations [38, 39, 73, 74]. Only the LM-G41R seems to be $\mathrm{N}$-terminal cleaved upon mitochondrial import $[38,73]$, possibly because of the enhanced conformational fluctuations caused by the mutation next to the MTS [43], which suggests that the MTS is not generally cleaved upon import of hAGT as observed for many other MTS-containing proteins. Interestingly, proteins containing short MTS are known to be imported by a mechanism where unfolding is rate-limiting $[75,76]$, and thus, the low kinetic stability of LM-G170R, LM-F152I and LM-G41R as apo-proteins [43, 48, $57,62]$ may speed up mitochondrial import of these mutants.
Besides the classical interaction between TOM20 mitochondrial receptors and N-terminal MTS, alternative mechanisms of mitochondrial import might be involved, such as presentation of internal targeting sequences in partially folded states to TOM20 or TOM70 receptors by molecular chaperones ([57, 77, 78]; see Figure 3$)$. Enhanced interaction of misfolded/partially folded PH1 variants with molecular chaperones seems to a common feature to many PH1 mutants $[34,48,50,57]$, and thus not only restricted to the mistargeting LM-F152I and LM-G170R variants. Some mutations, such as LM-I244T, have been studied by several groups using different cell lines (COS and $\mathrm{CHO}$ ) for heterologous gene expression, different cell culture media and PLP concentrations. Consequently, significant differences in mutant outcomes have been observed, showing either peroxisomal aggregation [34] or mitochondrial mistargeting [73], further supporting the view that different AGT load and variable capabilities of protein homeostasis networks may have a large impact on the final fate of PH1 causing mutants. Dissection the molecular details of these protein homeostasis defects is thus required to develop specific therapeutic strategies targeting different $\mathrm{PH} 1$ molecular mechanisms, possibly involving the cooperation of multiple proteostasis elements (Figure 3) including Hsp60, Hsp70 and Hsp90 machineries [34, 48, 50, 57].

A recent study on PTS1 octapeptides from 42 human peroxisomal proteins has shown that these sequences range over four orders of magnitude on their affinity for Pex5ppbd, and interestingly, the PTS1 corresponding to hAGT is the second weakest among this list [28]. A detailed analysis on protein expression levels for these peroxisomal proteins revealed a remarkable negative correlation between binding affinities and expression levels of cargo proteins, thus providing a mechanistic framework to generating a uniform population of Pex5p-cargo complexes necessary for proper peroxisomal biogenesis [28]. Pex5p-pbd binding studies of $\mathrm{PH} 1$ causing mutations G170R and V336D on the major allele [30] as well as some of the most common mutations on the minor allele, including G170R, I244T and F152I [48], revealed no changes in the molecular recognition of $\mathrm{PH} 1$ mutants by Pex5p, further supporting that folding defects are likely responsible for mitochondrial mistargeting and protein aggregation mechanisms in $\mathrm{PH} 1$.

3.2.2. Folding Defects Are Common to Mitochondrial Mistargeting and Aggregation in PH1: Targeting Protein Homeostasis Networks as Pharmacological Strategies for PH1. Early studies in liver samples from $\mathrm{PH} 1$ patients showed that the LM-G170R variant reduces the protein levels and activity and lead to mitochondrial mistargeting [39]. Further studies, expanded to LM-G41R/LM-F152I compound heterozygotes showed combined mitochondrial/peroxisomal location, intraperoxisomal aggregation and null activity [38]. The LM-F152I, LM-G170R and LM-I244T variants show reduced folding efficiency in both prokaryotic and eukaryotic systems $[40,48,57,73]$, while a milder effect is observed for LM polymorphic variant in these studies. These evidences clearly pointed to $\mathrm{PH} 1$ as conformational disease. However, up to 
date, the molecular details of $\mathrm{PH} 1$ protein homeostasis defects are mostly unknown.

We have recently initiated a comparative study on several $\mathrm{PH} 1$ variants that shows a positive correlation between apoAGT kinetic stability, interaction with molecular chaperones, and decrease solubility and total protein levels in transiently transfected CHO cells [48]. This seems to apply for either aggregation (LM-I244T) or mistargeting (LM-F152I and LMG170R) mechanisms. Accordingly, a recent study has shown that human AGT is capable of complementing yeast strains lacking endogenous yeast AGT in glycine-free medium [56], while LM-F152I and specially LM-I244T, but not LMG170R, reduced the complementation elicited by AGT LM. Complementation by $\mathrm{PH} 1$ mutants in this system closely correlated with steady-state expression levels, indicating that these mutants affect proper intracellular folding in vivo. More recently, using a novel stability reporter assay [60], a clear correlation between in vivo steady AGT levels and kinetic stability and yeast growth was found for LM-F152I, LMG170R and LM-I244T. Overall, these expression studies support a link between intracellular folding efficiency and kinetic stability of apo-AGT variants associated to mistargeting and aggregation mechanisms.

As we have mentioned in Section 3.2.1, the intracellular fate of AGT mutants depends on the experimental conditions of in vitro expression (LM-I244T is an excellent example; $[34,48,73])$. An interesting possibility to explain these differences is that different capacities of the protein homeostasis networks might determine the phenotype at the molecular level (aggregation versus mistargeting), a phenomena that has been described for disease penetrance even among isogenic individuals in animal models of several folding diseases [79]. These differences in folding capability might explain different phenotypic outcomes even among siblings sharing a given phenotype [2], and also possibly, the different response to pyridoxine supplementation among $\mathrm{PH} 1$ patients with the same phenotype $[63,64]$.

3.2.3. Interaction with Molecular Chaperones. Protein folding intermediates are commonly observed for proteins larger that 100 aminoacids, and these intermediates often require substantial structural reorganization upon interaction with molecular chaperones to reach the native conformation [80]. As we describe in Section 3.1.1, human AGT is known to populate (un)folding intermediates upon chemical denaturation, and these intermediates do not generally reactivate $[55,57]$ and also stably interact with several types of molecular chaperones ([34, 50, 57]; Figure 3$)$. Moreover, the strong interaction of several misfolding AGT mutants with molecular chaperones suggests that these chaperones might be important checkpoints in AGT folding and misfolding $[34,50,57]$. Thus, modulation of the interaction of partially folded states of AGT variants with molecular chaperones is a plausible approach for pharmacological intervention in PH1, as similarly described for other protein folding diseases [8183].

An important question is whether WT and $\mathrm{PH} 1$ causing mutants populate different folding/unfolding intermediates, or whether, kinetic/thermodynamic aspects of these folding intermediates and their interaction with the protein homeostasis networks [84-86] determine the final fate of AGT variants (mistargeting versus aggregation versus degradation). The structure/energetics of the TS for the rate limiting step of irreversible denaturation of WT and PH1 mutants is strikingly similar, suggesting a fine-tuning of the structure/energetics of this TS as determinant for the kinetic stability of PH1 mutants (unpublished observations). On the other hand, the structural properties of the chaperone competent MG state formed at mild acidic $\mathrm{pH}$ is similar for WT, LM and LM-G170R, but seems to accumulate in a Hsc70- or Hsp90-bound state upon expression in cell-free systems for LM-G170R [57]. Moreover, aggregation of AGT WT is prevented in vitro by its interaction along its folding pathway with Hsp40 in a partially folded state that resembles the acid-induced MG state (Figures 4(d)-4(e)), which may prevent AGT aggregation co- or post-translationally, thus delivering the polypeptide to the Hsc70 machinery $[80,86]$. Hsc70 machinery may represent the first essential folding checkpoint, as the interaction of these chaperones with Hsp40 proteins and nucleotide exchange factors [86] would determine the partitioning between correct folding and peroxisomal import, mitochondrial import or degradation (Figure 3). Transfer of cargo AGT from Hsp70 to Hsp90 might also occur through the Hsc70-Hsp90 organizing protein (HOP; [86]). Moreover, we have also described that overexpression of bacterial Hsp60 (GroEL; [50]) increase the recovery of LM-I244T in E. coli, forming stable complexes with GroEL. The characterization of the bound AGT LM-I244T by cryoelectron microscopy shows that AGT monomers display folded N- and C-terminal domains in an open conformation [50]. Overall, these studies suggest that the last steps in AGT folding involve domain docking and acquisition of quaternary structure that are crucial for AGT conformation of PH1 mutant. Thus, pharmacological modulation of the proteostasis pathways involved may rescue AGT function in PH1 patients.

\section{AGT as a Drugable Target for the Treatment of PH1}

Once the diagnosis of $\mathrm{PH1}$ is made, or even suspected, conservative measures should be initiated as soon as possible with the goal of preserving renal function. Once renal function is lost, the threat posed by progressive oxalate accumulation makes it necessary to perform liver and kidney transplantation, an aggressive treatment not free of difficulties, risks and limitations. Thus, current research aims at medical treatments for PH1.

High fluid intake has been proven to be effective in kidney stone diseases [87]. In $\mathrm{PH}$, the recommended fluid intake is at least $3 \mathrm{~L} / \mathrm{m}^{2}$ per day, and special care should be taken in situations of fluid losses (diarrhea, vomiting, and fever) or limited oral hydration (surgery), where i.v. fluid administration might be necessary to keep high urine flow. In addition, alkalinization of the urine with alkali citrate is implemented to reduce urinary $\mathrm{CaOx}$ saturation. 
Administration of pyridoxine hydrochloride was proposed as a conservative PH1-specific measure several decades ago. This form of treatment has been known to be associated with a decrease in urinary oxalate (UOx) in about $30 \%$ of $\mathrm{PH} 1$ patients $[88,89]$. Since vitamin B6 is very safe, with a small risk of sensory neurotoxicity as the main side effect, a trial of pyridoxine treatment should be performed in most cases, and particularly in patients with missense mutations. If responsive, patients should be treated until liver transplantation is performed, even if they are in ESRD undergoing haemodialysis. The recommended starting dose is $5 \mathrm{mg} / \mathrm{kg}$ per day, increasing in $5 \mathrm{mg} / \mathrm{kg}$ steps to a maximum of $20 \mathrm{mg} / \mathrm{kg}$ body weight per day [90]. Responsiveness is currently defined by $>30 \%$ decrease in UO $x$ excretion after a test period of a minimum of 3 months at maximum dose $[22,91]$. A subset of patients carrying one or two copies of G170R or F152I mutations have been shown to respond best to pyridoxine $[63,64]$.

In addition to the experimental data obtained in the last few years on the cellular and molecular consequences of various missense mutations, the empirical response of some PH1 patients to vitamin B6 treatment supports the view of AGT as a "drugable target" that deserves serious commitment to develop small molecules with enhanced chaperone capability or proteostatic effect.

As we describe in detail in Section 3.1.2, binding of PLP to AGT protein dramatically enhances its kinetic stability, particularly in variants of the minor haplotype, such as G170R and I244T [57]. The most relevant parameter to estimate the kinetic stability of properly folded cytosolic AGT dimers seems to be the half-life for irreversible denaturation of the apo-forms. Conversion of apo- to holo-AGT is a slow process that is catalyzed and regulated by specific enzyme systems $[57,65]$, and this conversion may trap the AGT in a folded and kinetically stable holo-form ready for peroxisomal import. This is especially relevant for $\mathrm{PH} 1$ missense mutations that are more destabilized in their apo-form than in their holo-form.

Building on this view of PLP as a natural kinetic stabilizer of AGT, it is reasonable to expect that pharmacological chaperones will be found that specifically bind to the AGT native state leading to thermodynamic and/or kinetic stabilizations. Ideally, they will have enhanced chaperone activity on mutant AGT variants, leading to more efficient conservative treatments for PH1. To move forward, a more precise undestanding of the mechanism of PLP kinetic "overstabilization" of missense mutations is needed. The precise molecular details of this overstabilization are under investigation.

Nevertheless, since protein stabilization can be searched by high-throughput screening of chemical and virtual libraries $[92,93]$, the possibility exists that potential pharmacological chaperones are found empirically. In some cases, pharmacological chaperones resemble known protein ligands or inhibitors, and their stabilizing effect may be enhanced by structure-based approaches [93, 94]. Pharmacological chaperones have been shown to correct protein misfolding in several genetic diseases and it seems likely that missense PH1 mutations can benefit from this approach also. In addition, targeting cellular systems responsible for PLP bioavailability (enzymes involved in PLP recycling and delivery; [65]) may represent a pharmacological approach to overcome mutation induced protein destabilization in $\mathrm{PH}$.

Chemical chaperones are small organic compounds which favor compact protein states over unfolded states through the so-called "solvophobic effect", which involves destabilizing interactions of the water/chaperone mixture with the polypeptide backbone [95]. Their beneficial effect on mutant AGT has been demonstrated in vitro [34, 43], although the high concentrations required for protein stabilization and the lack of specificity of the stabilizing effect make them poor candidates to move forward into preclinical investigation.

In the future, the complexity of AGT intracellular homeostasis will also be better understood. The macromolecular assemblies that assist AGT expression, folding, stability, intracellular trafficking and degradation include key components that are also drugable targets for conservative treatment of PH1. AGT native state kinetic stability [57, 62] and interactions of partially folded states with molecular chaperones such as Hsp40/DnaJ, Hsp60/GroEL, Hsc70 and Hsp90 [34, 48, 50,57] may be involved in the partition of AGT protein between correctly folded dimers and misfolding, with mitochondrial mistargeting, aggregation and degradation.

Pharmacological modulation of molecular chaperones is a promising therapeutic strategy in several conformational diseases [82]. However, the complexity of the molecular chaperone system in humans poses a significant challenge. The HSP70 machinery, for instance, includes at least 11 HSP70s, $41 \mathrm{~J}$-proteins and 13 nucleotide exchange factors (NEFs) [86]. In addition, mammalian J-proteins and NEF proteins are structurally and functionally complex, displaying additional functions beyond the canonical model. J-proteins are known to be involved in shunting client proteins for degradation, remodeling and partially unfolding client proteins, while the BAG (BCL2-associated athanogene) family of NEFs are also known to target client proteins for proteasomal degradation [86]. Thus, finding the ideal target and modulating it pharmacologically in just the right direction is a formidable challenge. As a first step, an exhaustive biochemical and cellular definition on all these targets is needed, in order to determine those responsible for mitochondrial mistargeting, aggregation and degradation of mutant AGT.

\section{Conflict of Interests}

The authors declare no conflict of interests.

\section{Acknowledgments}

The authors thank Professor Arturo Muga for the DnaJ expression clone. This work was supported by the Spanish ministry of Science and Innovation (RYC2009-04147 and CSD2009-00088 to Angel L. Pey, SAF2011-23933 to Eduardo Salido, and CSD2006-00015 and BFU2011-25384 to Armando Albert), Junta de Andalucia (P11CTS-7187 A.L.P.), and European Union (FP7-REGPOT-CT2012-31637-IMBRAIN to E.S.). 


\section{References}

[1] B. Hoppe, "An update on primary hyperoxaluria," Nature Reviews Nephrology, vol. 8, pp. 467-475, 2012.

[2] E. Salido, A. L. Pey, R. Rodriguez, and V. Lorenzo, "Primary hyperoxalurias: disorders of glyoxylate detoxification," Biochimica et Biophysica Acta, vol. 1822, no. 9, pp. 1453-1464, 2012.

[3] C. J. Danpure, "Primary hyperoxalurias," in The Metabolic and Molecular Bases of Inherited Disease, C. R. Scriver, A. L. Beaudet, W. S. Sly et al., Eds., vol. 2, pp. 3323-3367, McGraw-Hill, New York, NY, USA, 8th edition, 2001.

[4] M. Zylicz, T. Yamamoto, N. McKittrick, S. Sell, and C. Georgopoulos, "Purification and properties of the dnaJ replication protein of Escherichia coli," The Journal of Biological Chemistry, vol. 260, no. 12, pp. 7591-7598, 1985.

[5] W. Martin, "Evolutionary origins of metabolic compartmentalization in eukaryotes," Philosophical Transactions of the Royal Society B, vol. 365, no. 1541, pp. 847-855, 2010.

[6] R. J. A. Wanders and H. R. Waterham, "Biochemistry of mammalian peroxisomes revisited," Annual Review of Biochemistry, vol. 75, pp. 295-332, 2006.

[7] A. Rokka, V. D. Antonenkov, R. Soininen et al., "Pxmp2 is a channel-forming protein in mammalian peroxisomal membrane," PLoS ONE, vol. 4, no. 4, Article ID e5090, 2009.

[8] B. Cellini, M. Bertoldi, R. Montioli, A. Paiardini, and C. B. Voltattorni, "Human wild-type alanine:glyoxylate aminotransferase and its naturally occurring G82E variant: functional properties and physiological implications," Biochemical Journal, vol. 408, no. 1, pp. 39-50, 2007.

[9] J. Knight and R. P. Holmes, "Mitochondrial hydroxyproline metabolism: implications for primary hyperoxaluria," The American Journal of Nephrology, vol. 25, no. 2, pp. 171-175, 2005.

[10] J. Knight, J. Jiang, D. G. Assimos, and R. P. Holmes, "Hydroxyproline ingestion and urinary oxalate and glycolate excretion," Kidney International, vol. 70, no. 11, pp. 1929-1934, 2006.

[11] E. Adams and L. Frank, "Metabolism of proline and the hydroxyprolines," Annual Review of Biochemistry, vol. 49, pp. 1005-1061, 1980.

[12] R. P. Holmes and D. G. Assimos, "Glyoxylate synthesis, and its modulation and influence on oxalate synthesis," Journal of Urology, vol. 160, no. 5, pp. 1617-1624, 1998.

[13] J. M. Phang, C. A. Hu, and D. Valle, "Disorders in proline and hidroxyproline metabolism," in The Metabolic and Molecular Bases of Inherited Disease, C. R. Scriver, A. Beaudet, W. Sly, D. Valle, and B. Childs, Eds., pp. 1821-1838, McGraw-Hill, New York, NY, USA, 2001.

[14] P. Cochat, A. Deloraine, M. Rotily, F. Olive, I. Liponski, and N. Deries, "Epidemiology of primary hyperoxaluria type 1," Nephrology Dialysis Transplantation, vol. 10, supplement 8, pp. 3-7, 1995.

[15] N. Kopp and E. Leumann, "Changing pattern of primary hyperoxaluria in Switzerland," Nephrology Dialysis Transplantation, vol. 10, no. 12, pp. 2224-2227, 1995.

[16] C. S. van Woerden, J. W. Groothoff, R. J. A. Wanders, J. Davin, and F. A. Wijburg, "Primary hyperoxaluria type 1 in The Netherlands: prevalence and outcome," Nephrology Dialysis Transplantation, vol. 18, no. 2, pp. 273-279, 2003.

[17] V. Lorenzo, A. Alvarez, A. Torres, V. Torregrosa, D. Hernández, and E. Salido, "Presentation and role of transplantation in adult patients with type 1 primary hyperoxaluria and the I244T AGXT mutation: single-center experience," Kidney International, vol. 70, no. 6, pp. 1115-1119, 2006.
[18] J. Harambat, K. J. van Stralen, L. Espinosa et al., "Characteristics and outcomes of children with primary oxalosis requiring renal replacement therapy," Clinical Journal of the American Society of Nephrology, vol. 7, no. 3, pp. 458-465, 2012.

[19] A. A. Al-Eisa, M. Samhan, and M. Naseef, "End-stage renal disease in Kuwaiti children: an 8-year experience," Transplantation Proceedings, vol. 36, no. 6, pp. 1788-1791, 2004.

[20] A. Kamoun and R. Lakhoua, "End-stage renal disease of the Tunisian child: epidemiology, etiologies, and outcome," Pediatric Nephrology, vol. 10, no. 4, pp. 479-482, 1996.

[21] C. J. Danpure and P. R. Jennings, "Peroxisomal alanine:glyoxylate aminotransferase deficiency in primary hyperoxaluria type I," FEBS Letters, vol. 201, no. 1, pp. 20-24, 1986.

[22] E. Leumann and B. Hoppe, "The primary hyperoxalurias," Journal of the American Society of Nephrology, vol. 12, no. 9, pp. 1986-1993, 2001.

[23] K. Nishiyama, G. Berstein, T. Oda, and A. Ichiyama, "Cloning and nucleotide sequence of cDNA encoding human liver serinepyruvate aminotransferase," European Journal of Biochemistry, vol. 194, no. 1, pp. 9-18, 1990.

[24] Y. Takada, N. Kaneko, H. Esumi, P. E. Purdue, and C. J. Danpure, "Human peroxisomal L-alanine:glyoxylate aminotransferase. Evolutionary loss of a mitochondrial targeting signal by point mutation of the initiation codon," Biochemical Journal, vol. 268, no. 2, pp. 517-520, 1990.

[25] T. Oda, T. Funai, and A. Ichiyama, "Generation from a single gene of two mRNAs that encode the mitochondrial and peroxisomal serine:pyruvate aminotransferase of rat liver," The Journal of Biological Chemistry, vol. 265, no. 13, pp. 7513-7519, 1990.

[26] P. E. Purdue, M. J. Lumb, M. Fox et al., "Characterization and chromosomal mapping of a genomic clone encoding human alanine: glyoxylate aminotransferase," Genomics, vol. 10, no. 1, pp. 34-42, 1991.

[27] X. Zhang, S. M. Roe, Y. Hou et al., "Crystal structure of alanine:glyoxylate aminotransferase and the relationship between genotype and enzymatic phenotype in primary hyperoxaluria type 1," Journal of Molecular Biology, vol. 331, no. 3, pp. 643-652, 2003.

[28] D. Ghosh and J. M. Berg, "A proteome-wide perspective on peroxisome targeting signal 1(PTS1)-Pex5p affinities," Journal of the American Chemical Society, vol. 132, no. 11, pp. 3973-3979, 2010.

[29] P. A. J. Huber, G. M. Birdsey, M. J. Lumb et al., "Peroxisomal import of human alanine:glyoxylate aminotransferase requires ancillary targeting information remote from its C terminus," The Journal of Biological Chemistry, vol. 280, no. 29, pp. 2711127120, 2005.

[30] K. Fodor, J. Wolf, R. Erdmann, W. Schliebs, and M. Wilmanns, "Molecular requirements for peroxisomal targeting of alanineglyoxylate aminotransferase as an essential determinant in primary hyperoxaluria type 1," PLoS Biology, vol. 10, no. 4, Article ID e1001309, 2012.

[31] E. L. Williams, C. Acquaviva, A. Amoroso et al., "Primary hyperoxaluria type 1: update and additional mutation analysis of the AGXT gene," Human Mutation, vol. 30, no. 6, pp. 910917, 2009.

[32] E. Williams and G. Rumsby, "Selected exonic sequencing of the AGXT gene provides a genetic diagnosis in $50 \%$ of patients with primary hyperoxaluria type I," Clinical Chemistry, vol. 53, no. 7, pp. 1216-1221, 2007. 
[33] C. J. Danpure and G. Rumsby, "Molecular aetiology of primary hyperoxaluria and its implications for clinical management," Expert Reviews in Molecular Medicine, vol. 6, no. 1, pp. 1-16, 2004.

[34] A. Santana, E. Salido, A. Torres, and L. J. Shapiro, "Primary hyperoxaluria type 1 in the Canary Islands: a conformational disease due to I244T mutation in the P11L-containing alanine:glyoxylate aminotransferase," Proceedings of the National Academy of Sciences of the United States of America, vol. 100, no. 12, pp. 7277-7282, 2003.

[35] C. J. Danpure, P. R. Jennings, P. Fryer, P. E. Purdue, and J. Allsop, "Primary hyperoxaluria type 1: genotypic and phenotypic heterogeneity," Journal of Inherited Metabolic Disease, vol. 17, no. 4, pp. 487-499, 1994.

[36] K. Nishiyama, T. Funai, S. Yokota, and A. Ichiyama, "ATP-dependent degradation of a mutant serine:pyruvate/alanine:glyoxylate aminotransferase in a primary hyperoxaluria type 1 case," Journal of Cell Biology, vol. 123, no. 5, pp. 1237-1248, 1993.

[37] C. J. Danpure, P. J. Cooper, P. J. Wise, and P. R. Jennings, “An enzyme trafficking defect in two patients with primary hyperoxaluria type 1: peroxisomal analine/glyoxylate aminotransferase rerouted to mitochondria," Journal of Cell Biology, vol. 108, no. 4, pp. 1345-1352, 1989.

[38] C. J. Danpure, P. E. Purdue, P. Fryer et al., "Enzymological and mutational analysis of a complex primary hyperoxaluria type I phenotype involving alanine:glyoxylate aminotransferase peroxisome-to-mitochondrion mistargeting and intraperoxisomal aggregation," The American Journal of Human Genetics, vol. 53, no. 2, pp. 417-432, 1993.

[39] P. E. Purdue, Y. Takada, and C. J. Danpure, "Identification of mutations associated with peroxisome-to-mitochondrian mistargeting of alanine/glyoxylate aminotransferase in primary hyperoxaluria Type 1," Journal of Cell Biology, vol. 111, no. 6 I, pp. 2341-2351, 1990.

[40] M. J. Lumb and C. J. Danpure, "Functional synergism between the most common polymorphism in human alanine:glyoxylate aminotransferase and four of the most common diseasecausing mutations," The Journal of Biological Chemistry, vol. 275, no. 46, pp. 36415-36422, 2000.

[41] C. J. Danpure, "Primary hyperoxaluria type 1: AGT mistargeting highlights the fundamental differences between the peroxisomal and mitochondrial protein import pathways," Biochimica et Biophysica Acta, vol. 1763, no. 12, pp. 1776-1784, 2006.

[42] L. M. Luheshi and C. M. Dobson, "Bridging the gap: from protein misfolding to protein misfolding diseases," FEBS Letters, vol. 583, no. 16, pp. 2581-2586, 2009.

[43] B. Cellini, R. Montioli, A. Paiardini et al., "Molecular defects of the glycine 41 variants of alanine glyoxylate aminotransferase associated with primary hyperoxaluria type I," Proceedings of the National Academy of Sciences of the United States of America, vol. 107, no. 7, pp. 2896-2901, 2010.

[44] B. Cellini, R. Montioli, and C. B. Voltattorni, "Human liver peroxisomal alanine:glyoxylate aminotransferase: characterization of the two allelic forms and their pathogenic variants," Biochimica et Biophysica Acta, vol. 1814, no. 11, pp. 1577-1584, 2011.

[45] D. Pirulli, M. Marangella, and A. Amoroso, "Primary hyperoxaluria: genotype-phenotype correlation," Journal of Nephrology, vol. 16, no. 2, pp. 297-309, 2003.

[46] G. Rumsby, E. Williams, and M. Coulter-Mackie, "Evaluation of mutation screening as a first line test for the diagnosis of the primary hyperoxalurias," Kidney International, vol. 66, no. 3, pp. 959-963, 2004.

[47] B. Hoppe, C. J. Danpure, G. Rumsby et al., "A vertical (pseudodominant) pattern of inheritance in the autosomal recessive disease primary hyperoxaluria type 1: lack of relationship between genotype, enzymic phenotype, and disease severity," The American Journal of Kidney Diseases, vol. 29, no. 1, pp. 3644, 1997.

[48] N. Mesa-Torres, I. Fabelo-Rosa, D. Riverol et al., "The role of protein denaturation energetics and molecular chaperones in the aggregation and mistargeting of mutants causing primary hyperoxaluria type I," PLOS ONE. In press.

[49] S. Djordjevic, X. Zhang, M. Bartlam, S. Ye, Z. Rao, and C. J. Danpure, "Structural implications of a G170R mutation of alanine:glyoxylate aminotransferase that is associated with peroxisome-to-mitochondrion mistargeting," Acta Crystallographica F, vol. 66, no. 3, pp. 233-236, 2010.

[50] A. Albert, C. Yunta, R. Arranz et al., "Structure of GroEL in complex with an early folding intermediate of alanine glyoxylate aminotransferase," The Journal of Biological Chemistry, vol. 285, no. 9, pp. 6371-6376, 2010.

[51] W. E. Balch, R. I. Morimoto, A. Dillin, and J. W. Kelly, "Adapting proteostasis for disease intervention," Science, vol. 319, no. 5865, pp. 916-919, 2008.

[52] E. T. Powers, R. I. Morimoto, A. Dillin, J. W. Kelly, and W. E. Balch, "Biological and chemical approaches to diseases of proteostasis deficiency," Annual Review of Biochemistry, vol. 78, pp. 959-991, 2009.

[53] F. U. Hartl, A. Bracher, and M. Hayer-Hartl, "Molecular chaperones in protein folding and proteostasis," Nature, vol. 475, no. 7356, pp. 324-332, 2011.

[54] R. I. Morimoto, "Proteotoxic stress and inducible chaperone networks in neurodegenerative disease and aging," Genes and Development, vol. 22, no. 11, pp. 1427-1438, 2008.

[55] B. Cellini, A. Lorenzetto, R. Montioli, E. Oppici, and C. B. Voltattorni, "Human liver peroxisomal alanine:glyoxylate aminotransferase: different stability under chemical stress of the major allele, the minor allele, and its pathogenic G170R variant," Biochimie, vol. 92, no. 12, pp. 1801-1811, 2010.

[56] E. D. Hopper, A. M. C. Pittman, M. C. Fitzgerald, and C. L. Tucker, "In vivo and in vitro examination of stability of primary hyperoxaluria-associated human alanine:glyoxylate aminotransferase," The Journal of Biological Chemistry, vol. 283, no. 45, pp. 30493-30502, 2008.

[57] A. L. Pey, E. Salido, and J. M. Sanchez-Ruiz, "Role of low native state kinetic stability and interaction of partially unfolded states with molecular chaperones in the mitochondrial protein mistargeting associated with primary hyperoxaluria," Amino Acids, vol. 41, no. 5, pp. 1233-1245, 2011.

[58] M. B. Coulter-Mackie, Q. Lian, and S. G. Wong, "Overexpression of human alanine:glyoxylate aminotransferase in Escherichia coli: renaturation from guanidine- $\mathrm{HCl}$ and affinity for pyridoxal phosphate co-factor," Protein Expression and Purification, vol. 41, no. 1, pp. 18-26, 2005.

[59] E. Oppici, R. Montioli, A. Lorenzetto, S. Bianconi, C. B. Voltattorni, and B. Cellini, "Biochemical analyses are instrumental in identifying the impact of mutations on holo and/or apoforms and on the region(s) of alanine:glyoxylate aminotransferase variants associated with primary hyperoxaluria type I," Molecular Genetics and Metabolism, vol. 105, no. 1, pp. 132-140, 2012. 
[60] A. M. Pittman, M. D. Lage, V. Poltoratsky et al., "Rapid profiling of disease alleles using a tunable reporter of protein misfolding," Genetics, vol. 192, no. 3, pp. 831-842, 2012.

[61] J. M. Sanchez-Ruiz, "Protein kinetic stability," Biophysical Chemistry, vol. 148, no. 1-3, pp. 1-15, 2010.

[62] B. Cellini, R. Montioli, A. Paiardini, A. Lorenzetto, and C. B. Voltattorni, "Molecular insight into the synergism between the minor allele of human liver peroxisomal alanine:glyoxylate aminotransferase and the F1521 mutation," The Journal of Biological Chemistry, vol. 284, no. 13, pp. 8349-8358, 2009.

[63] C. G. Monico, S. Rossetti, J. B. Olson, and D. S. Milliner, "Pyridoxine effect in type I primary hyperoxaluria is associated with the most common mutant allele," Kidney International, vol. 67, no. 5, pp. 1704-1709, 2005.

[64] C. S. van Woerden, J. W. Groothoff, F. A. Wijburg, C. Annink, R. J. A. Wanders, and H. R. Waterham, "Clinical implications of mutation analysis in primary hyperoxaluria type 1," Kidney International, vol. 66, no. 2, pp. 746-752, 2004.

[65] M. L. di Salvo, R. Contestabile, and M. K. Safo, "Vitamin B6 salvage enzymes: mechanism, structure and regulation," Biochimica et Biophysica Acta, vol. 1814, no. 11, pp. 1597-1608, 2011.

[66] C. Park and S. Marqusee, "Probing the high energy states in proteins by proteolysis," Journal of Molecular Biology, vol. 343, no. 5, pp. 1467-1476, 2004.

[67] M. B. Coulter-Mackie and Q. Lian, "Consequences of missense mutations for dimerization and turnover of alanine:glyoxylate aminotransferase: study of a spectrum of mutations," Molecular Genetics and Metabolism, vol. 89, no. 4, pp. 349-359, 2006.

[68] M. B. Coulter-Mackie and Q. Lian, "Partial trypsin digestion as an indicator of mis-folding of mutant alanine:glyoxylate aminotransferase and chaperone effects of specific ligands. Study of a spectrum of missense mutants," Molecular Genetics and Metabolism, vol. 94, no. 3, pp. 368-374, 2008.

[69] G. Tur-Arlandis, D. Rodriguez-Larrea, B. Ibarra-Molero, and J. M. Sanchez-Ruiz, "Proteolytic scanning calorimetry: a novel methodology that probes the fundamental features of protein kinetic stability," Biophysical Journal, vol. 98, no. 6, pp. L12-L14, 2010.

[70] C. J. Danpure, "Variable peroxisomal and mitochondrial targeting of alanine: glyoxylate aminotransferase in mammalian evolution and disease," BioEssays, vol. 19, no. 4, pp. 317-326, 1997.

[71] J. D. Holbrook and C. J. Danpure, "Molecular basis for the dual mitochondrial and cytosolic localization of alanine:glyoxylate aminotransferase in amphibian liver cells," The Journal of Biological Chemistry, vol. 277, no. 3, pp. 2336-2344, 2002.

[72] G. M. Birdsey, J. Lewin, J. D. Holbrook, V. R. Simpson, A. A. Cunningham, and C. J. Danpure, "A comparative analysis of the evolutionary relationship between diet and enzyme targeting in bats, marsupials and other mammals," Proceedings of the Royal Society B, vol. 272, no. 1565, pp. 833-840, 2005.

[73] S. Fargue, J. Lewin, G. Rumsby, and C. J. Danpure, "Four of the most common mutations in primary hyperoxaluria type 1 unmask the cryptic mitochondrial targeting sequence of alanine:glyoxylate aminotransferase encoded by the polymorphic minor allele," The Journal of Biological Chemistry, vol. 288, no. 4, pp. 2475-2484, 2013.

[74] P. E. Purdue, J. Allsop, G. Isaya, L. E. Rosenberg, and C. J. Danpure, "Mistargeting of peroxisomal L-alanine:glyoxylate aminotransferase to mitochondria in primary hyperoxaluria patients depends upon activation of a cryptic mitochondrial targeting sequence by a point mutation," Proceedings of the National Academy of Sciences of the United States of America, vol. 88, no. 23, pp. 10900-10904, 1991.

[75] A. Matouschek, A. Azem, K. Ratliff, B. S. Glick, K. Schmid, and G. Schatz, "Active unfolding of precursor proteins during mitochondrial protein import," EMBO Journal, vol. 16, no. 22, pp. 6727-6736, 1997.

[76] A. J. Wilcox, J. Choy, C. Bustamante, and A. Matouschek, "Effect of protein structure on mitochondrial import," Proceedings of the National Academy of Sciences of the United States of America, vol. 102, no. 43, pp. 15435-15440, 2005.

[77] J. C. Young, N. J. Hoogenraad, and F. U. Hartl, "Molecular chaperones Hsp90 and Hsp70 deliver preproteins to the mitochondrial import receptor Tom70," Cell, vol. 112, no. 1, pp. 41-50, 2003.

[78] H. Yamamoto, K. Fukui, H. Takahashi et al., "Roles of Tom70 in import of presequence-containing mitochondrial proteins," The Journal of Biological Chemistry, vol. 284, no. 46, pp. 3163531646, 2009.

[79] M. O. Casanueva, A. Burga, and B. Lehner, "Fitness trade-offs and environmentally induced mutation buffering in isogenic $C$. elegans," Science, vol. 335, no. 6064, pp. 82-85, 2012.

[80] F. U. Hartl and M. Hayer-Hartl, "Converging concepts of protein folding in vitro and in vivo," Nature Structural and Molecular Biology, vol. 16, no. 6, pp. 574-581, 2009.

[81] D. W. Neef, M. L. Turski, and D. J. Thiele, "Modulation of heat shock transcription factor 1 as a therapeutic target for small molecule intervention in neurodegenerative disease," PLoS Biology, vol. 8, no. 1, Article ID e1000291, 2010.

[82] D. S. T. Ong and J. W. Kelly, "Chemical and/or biological therapeutic strategies to ameliorate protein misfolding diseases," Current Opinion in Cell Biology, vol. 23, no. 2, pp. 231-238, 2011.

[83] B. Calamini, M. C. Silva, F. Madoux et al., "Small-molecule proteostasis regulators for protein conformational diseases," Nature Chemical Biology, vol. 8, no. 2, pp. 185-196, 2012.

[84] S. Prakash and A. Matouschek, "Protein unfolding in the cell," Trends in Biochemical Sciences, vol. 29, no. 11, pp. 593-600, 2004.

[85] A. J. McClellan, S. Tam, D. Kaganovich, and J. Frydman, "Protein quality control: chaperones culling corrupt conformations," Nature Cell Biology, vol. 7, no. 8, pp. 736-741, 2005.

[86] H. H. Kampinga and E. A. Craig, "The HSP70 chaperone machinery: J proteins as drivers of functional specificity," Nature Reviews Molecular Cell Biology, vol. 11, no. 8, pp. 579-592, 2010.

[87] L. Borghi, T. Meschi, F. Amato, A. Briganti, A. Novarini, and A. Giannini, "Urinary volume, water and recurrences in idiopathic calcium nephrolithiasis: a 5-year randomized prospective study," Journal of Urology, vol. 155, no. 3, pp. 839843, 1996.

[88] D. A. Gibbs and R. W. Watts, "The action of pyridoxine in primary hyperoxaluria," Clinical science, vol. 38 , no. 2, pp. 277286, 1970.

[89] R. W. E. Watts, N. Veall, P. Purkiss, M. A. Mansell, and E. F. Haywood, "The effect of pyridoxine on oxalate dynamics in three cases of primary hyperoxaluria (with glycollic aciduria)," Clinical Science, vol. 69, no. 1, pp. 87-90, 1985.

[90] B. Hoppe, K. Latta, C. von Schnakenburg, and M. J. Kemper, "Primary hyperoxaluria-the German experience," The American Journal of Nephrology, vol. 25, no. 3, pp. 276-281, 2005.

[91] B. Hoppe, B. B. Beck, and D. S. Milliner, "The primary hyperoxalurias," Kidney International, vol. 75, no. 12, pp. 12641271, 2009. 
[92] A. L. Pey, M. Ying, N. Cremades et al., "Identification of pharmacological chaperones as potential therapeutic agents to treat phenylketonuria," Journal of Clinical Investigation, vol. 118, no. 8, pp. 2858-2867, 2008.

[93] J. Underhaug, O. Aubi, and A. Martinez, "Phenylalanine hydroxylase misfolding and pharmacological chaperones," Current Topics in Medicinal Chemistry, vol. 12, no. 22, pp. 25342545, 2012.

[94] S. Connelly, S. Choi, S. M. Johnson, J. W. Kelly, and I. A. Wilson, "Structure-based design of kinetic stabilizers that ameliorate the transthyretin amyloidoses," Current Opinion in Structural Biology, vol. 20, no. 1, pp. 54-62, 2010.

[95] D. W. Bolen and G. D. Rose, "Structure and energetics of the hydrogen-bonded backbone in protein folding," Annual Review of Biochemistry, vol. 77, pp. 339-362, 2008. 

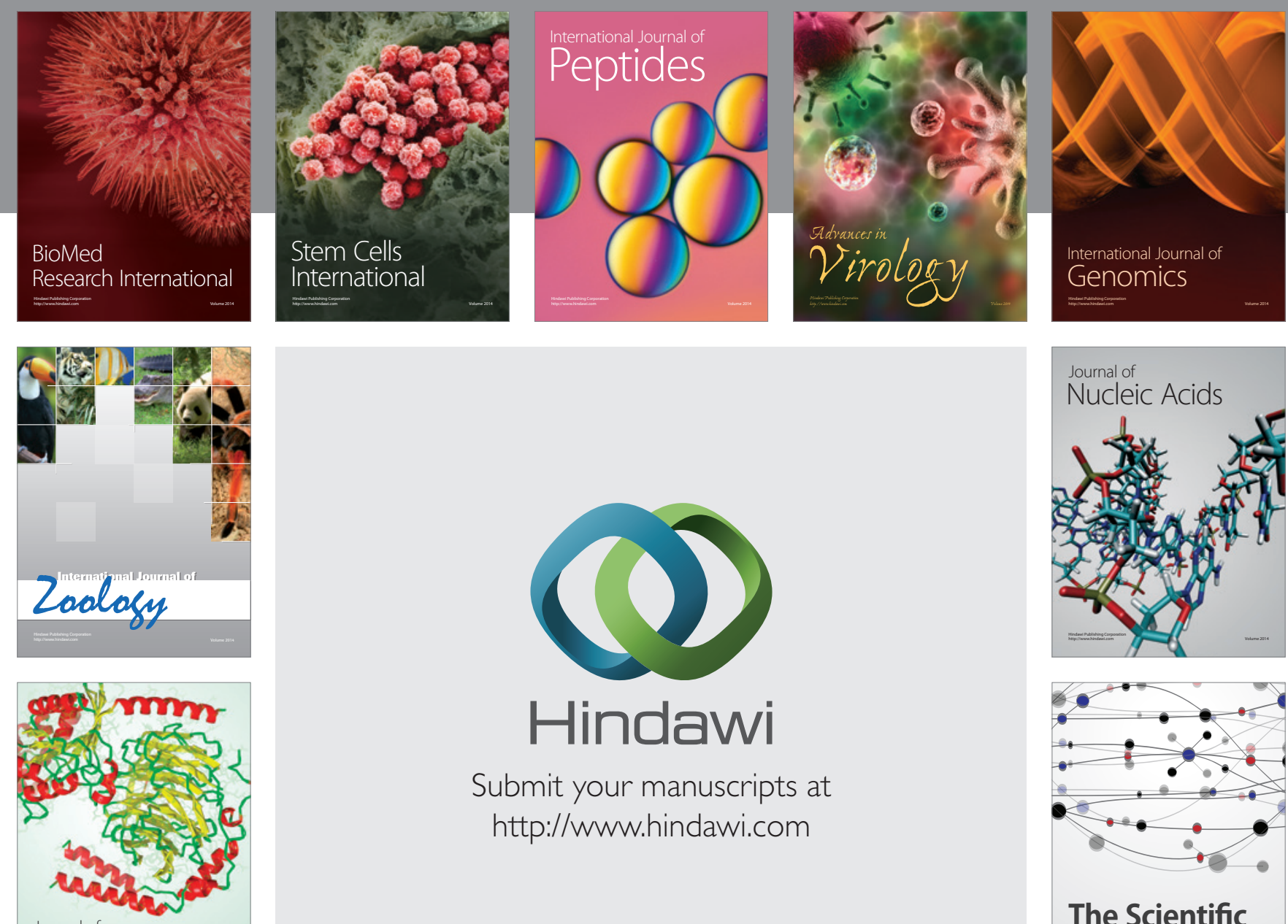

Submit your manuscripts at

http://www.hindawi.com

Journal of
Signal Transduction
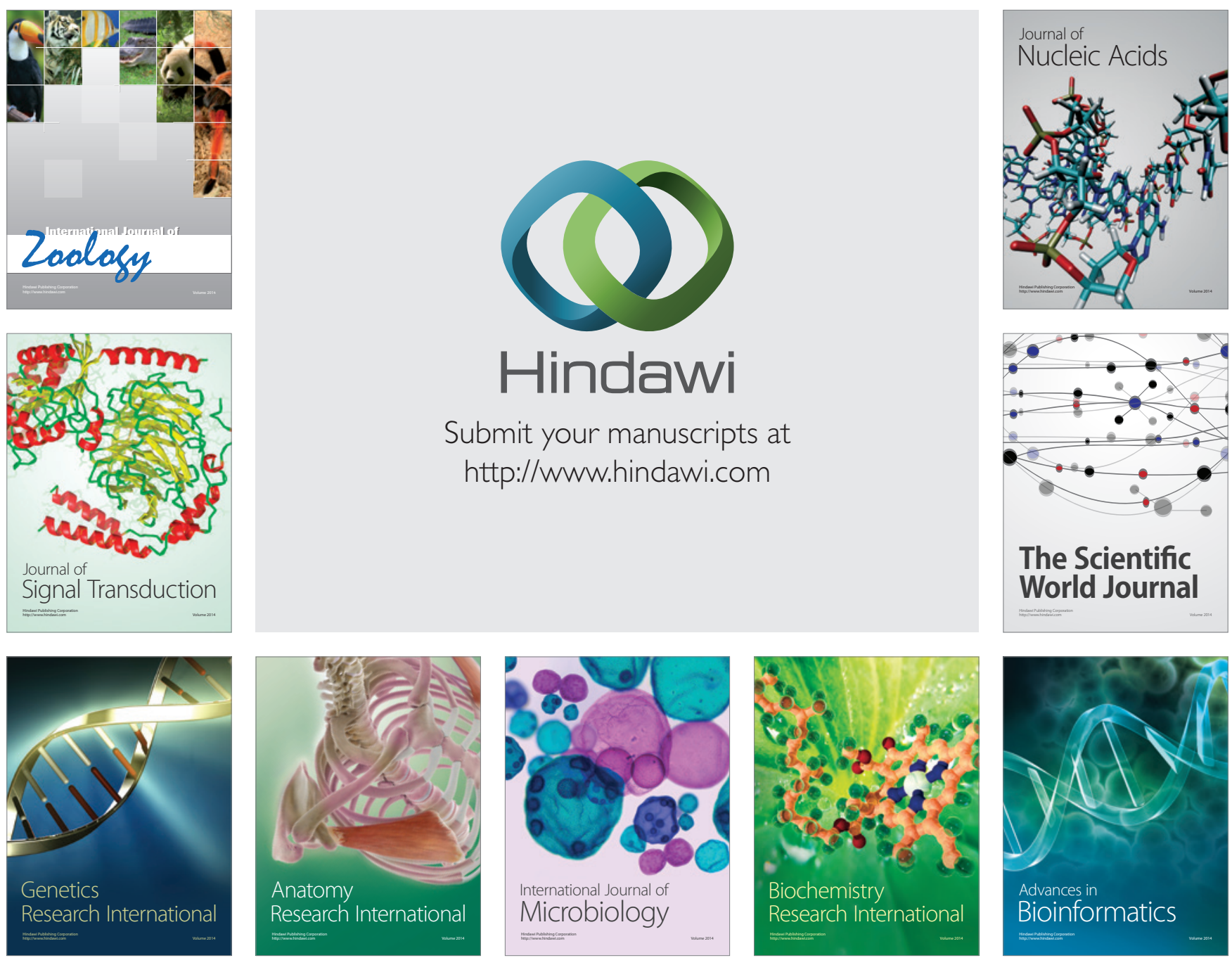

The Scientific World Journal
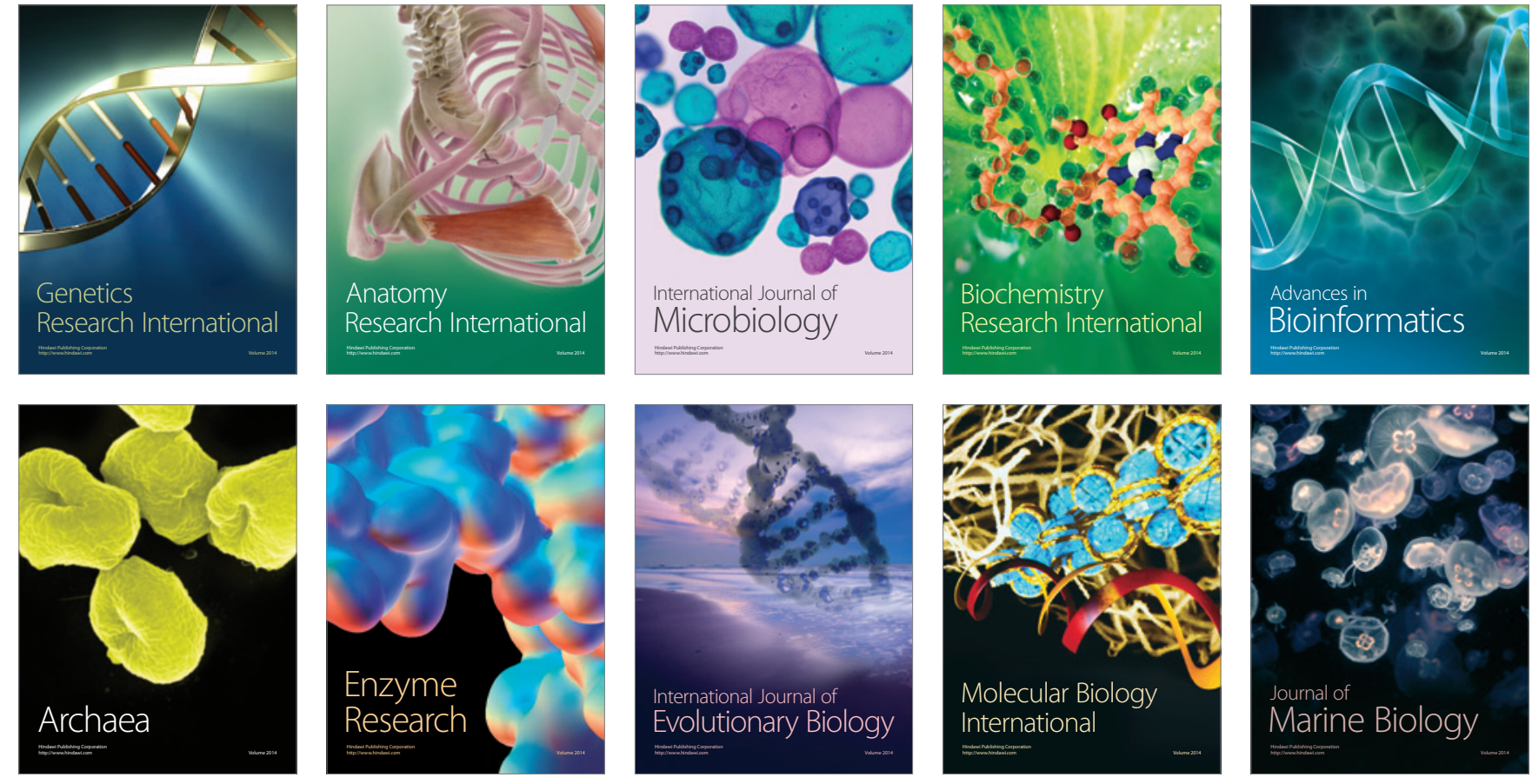